\title{
CNN-based Method for Segmenting Tree Bark Surface Singularites
}

\author{
Florian Delconte ${ }^{1}$, Phuc Ngo ${ }^{1}$, Bertrand Kerautret ${ }^{2}$, Isabelle Debled-Rennesson ${ }^{1}$, \\ Van-Tho Nguyen ${ }^{3}$, Thiery Constant ${ }^{4}$ \\ ${ }^{1}$ Université de Lorraine, LORIA, ADAGIo, Nancy, France (florian.delconte@loria.fr) \\ ${ }^{2}$ Université Lumière Lyon 2, LIRIS, Imagine, Lyon, France \\ ${ }^{3}$ Department of Applied Geomatics, Centre d'applications et de recherche en télédétection, \\ Université de Sherbrooke, Sherbrooke, Canada \\ ${ }^{4}$ Université de Lorraine, AgroParisTech, INRAE, SILVA, Nancy, France \\ Communicated by Julie Digne Demo edited by Florian Delconte
}

\begin{abstract}
The analysis of trunk shape and, in particular, the geometric structures on the bark surface are of main interest for different applications linked to the wood industry or biological studies. Bark singularities are often external records of the history of the development of internal elements. The actors of the forest sector grade the trees by considering these singularities through standards. In this paper, we propose a method using terrestrial LiDAR data to automatically segment singularities on tree surfaces. It is based on the construction of a relief map combined with a convolutional neural network. The algorithms and the source code are available with an online demonstration allowing to test the defect detection without any software installation.
\end{abstract}

\section{Source Code}

The reviewed source code and documentation associated to the proposed algorithms are available from the web page of this article ${ }^{1}$. The correspondences between algorithms and source codes are pointed out, and compilation with usage instructions are included in the README.md file of the archive.

\section{Supplementary Material}

The reference dataset, to be used for further comparisons, is provided with the article and peerreviewed. The (non peer-reviewed) instructions to generate the learned model are provided as supplementary material on the associated Github repository ${ }^{2}$.

Keywords: tree bark surface analysis; singularity segmentation; relief map; LiDAR; mesh centerline; neural network; U-Net

\footnotetext{
${ }^{1}$ https://doi.org/10.5201/ipol.2022.369

${ }^{2}$ https://github.com/FlorianDelconte/uNet_reliefmap
} 


\section{Introduction}

The development of trees is under the control of genetic factors in interaction with different components of their environment. This development results from different meristems producing living tissues, reacting to the ambient conditions, and producing also new meristems throughout the life of the tree. Thus, buds, leaves, branches, wood and bark result from such biological processes. This ability is essential for the tree to cope with the constraints evolving during its whole life, for adapting its structure as it grows and looks for light. Of course, these constraints are dependent on climatic conditions and on competition for light, water and nutrients with the tree's neighbors. They can also be linked to events such as frost cracking longitudinally the log, or strong wind breaking branches, or activity of insects or animals, or forester's action. This ability produces efficient elements for photosynthesis like branches supporting leaves, but also less efficient elements like burls in Figure 1 (a). It also enables the tree to self-repair when a branch disappears as illustrated in Figure 1 (b,c,d), or when a frost crack occurs in Figure 1 (f), or when the bark is damaged during the harvesting of neighbors, as in Figure 1 (e). The result is an alteration of the internal wood structure, which can be problematic for wood use as a material, but it also coincides with a modification of the bark structure. The later provides information about the size of the internal singularity and eventually about its developmental trajectory, especially in case of branches' scars.

The goal of this work is to automatically detect singularities (or "defects" in industrial context) located on the log surface. The detection of these singularities is an important step to determine the commercial value of trees and to optimize the processes that transform them into wood. It is not an easy task since each type of singularity presents many inter and intra species geometrical variations.

In previous work [1], we proposed a method based on the construction of a relief map combined with a convolutional neural network (CNN) allowing the accurate segmentation of singularities from input LiDAR 3D points of tree bark surface. In this paper, the algorithms of the proposed method are detailed together with the correspondence between algorithms and implementations. An online demonstration for testing the proposed method is available ${ }^{3}$. Moreover, we improve the generation of the relief map allowing to process input meshes of the partial tree bark surface. Finally, new experiments are presented in Section 4. A discussion of different parameters influence and limits for generating the relief map is given in Section 6. Before describing the segmentation method and algorithms, we first briefly give an overview of the main related works in the following section.

\section{Related Works}

In general, singularities (or defects) on the surface of a tree bark are distinguishable from the rest of the trunk because they form a local variation of relief. Singularity detection methods considered in this paper can be classified into two groups: the classical methods and the deep learning based methods.

\subsection{Classical Methods}

In 2013, Kretschmer et al. [8] proposed a semi-automatic method for measuring branch scars on the surface of tree trunks using terrestrial laser scanning (TLS, also called topographic LiDAR) data. This method is based on a cylinder fitting along the main axis of the trunk. From this cylinder, a distance map is generated. This 2D map allows the authors to measure manually the dimensions of the branch scars on the tree surface. However, due to the non-cylindricity of the trunk, long vertical traces are observed on the distance map (see Figure 2).

\footnotetext{
${ }^{3}$ https://ipolcore.ipol.im/demo/clientApp/demo.html?id=369
} 


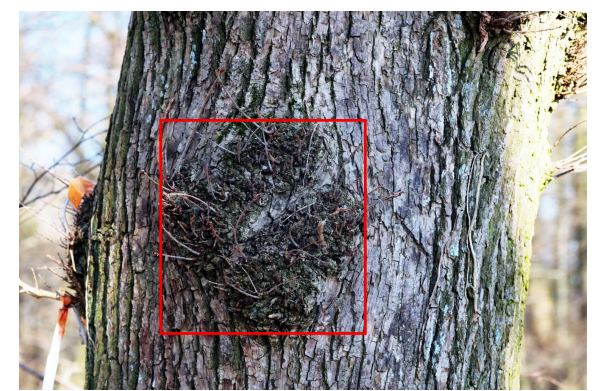

(a) Burl (Oak)

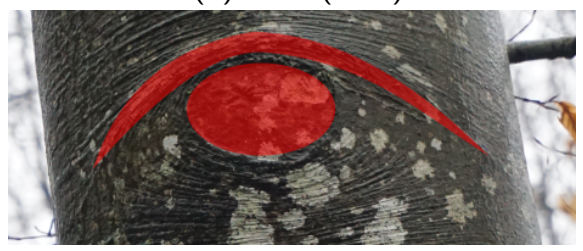

(b) Branch scar (Beech)

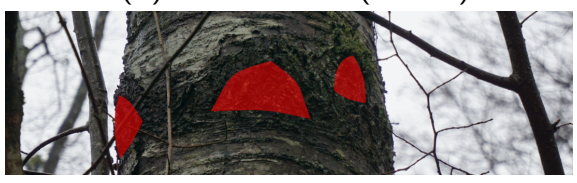

(c) Branch scar (cherry tree)

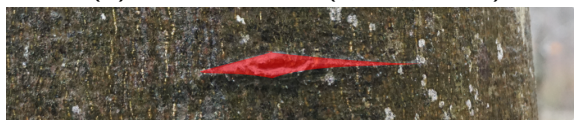

(d) Little branch scar (Beech)

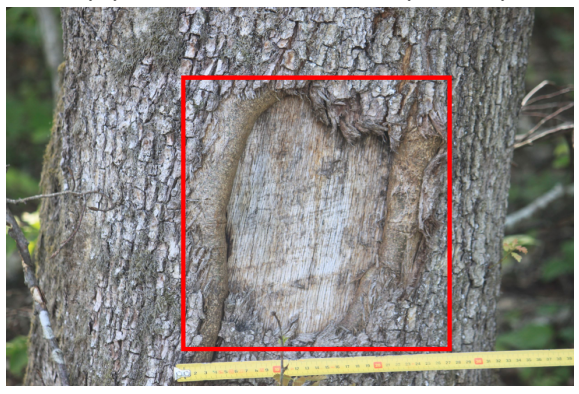

(e) Damaged bark (Oak)

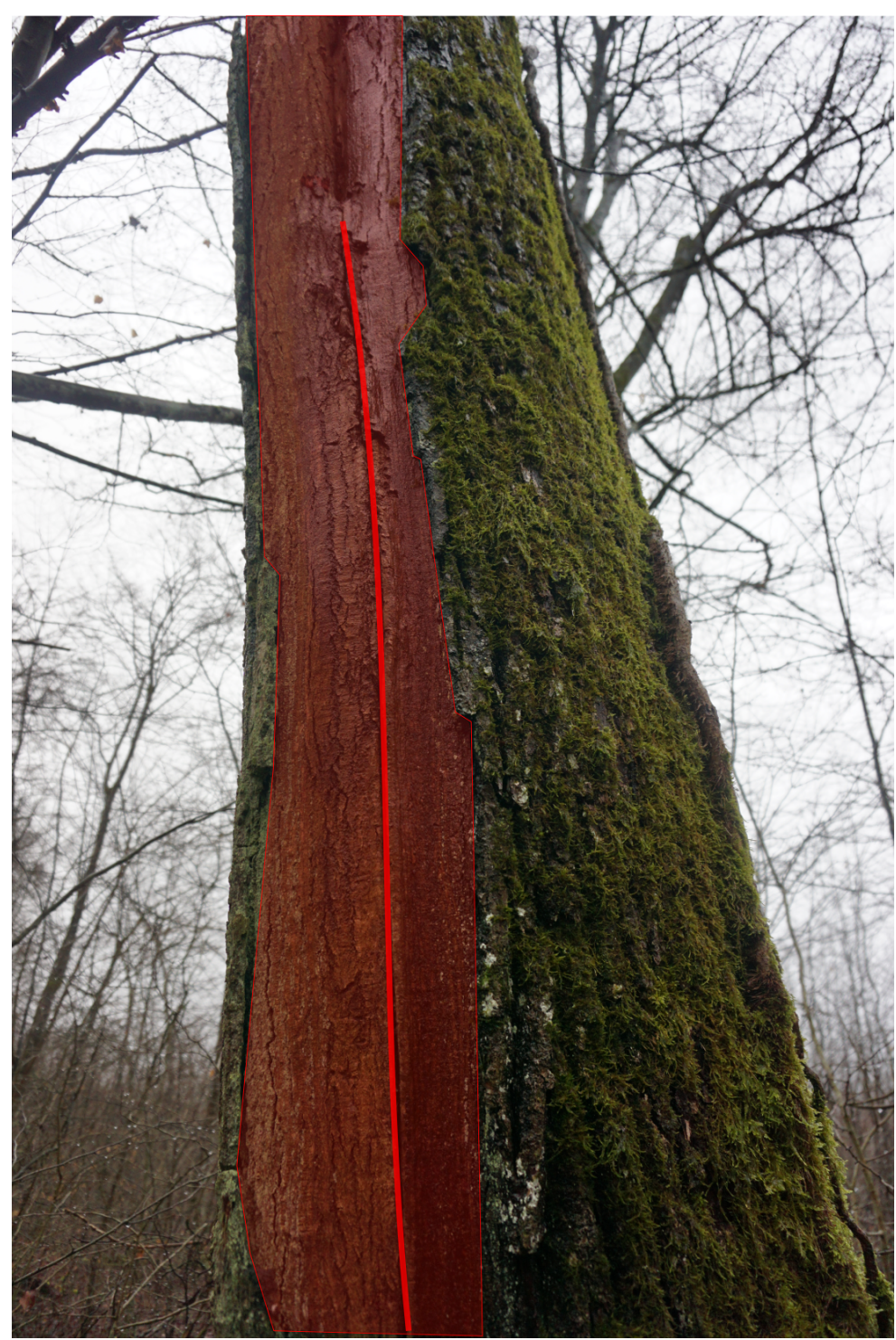

(f) Frost crack (Oak)

Figure 1: Examples of tree bark surface singularities (also called defects): branch scar, burl and frost crack. The singularities are highlighted in red. 


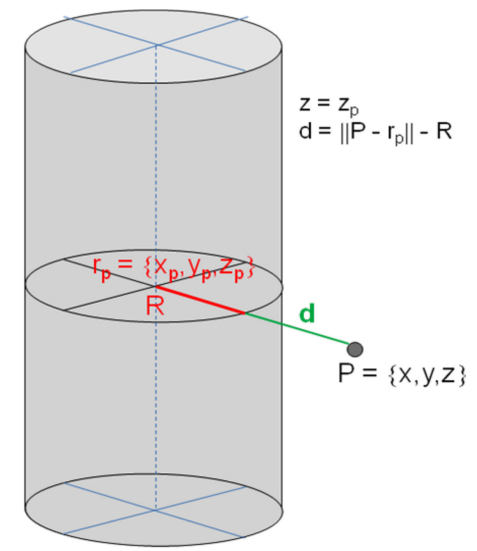

(a) Cylinder fitting

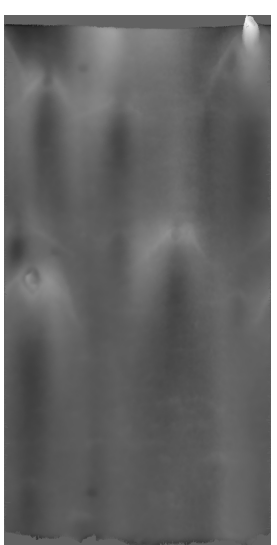

(b) Distance map

Figure 2: Singularity detection based on cylinder fitting: (a) the cylinder fitted from the point cloud, with $d$ the distance used to compute the distance map. (b) Illustration extracted from [8].

Based on another tree bark surface representation, Nguyen et al. [11] proposed an automatic singularity segmentation method. This method takes as input a mesh reconstructed from TLS data. It builds the centerline [5] of the trunk. This centerline allows to compute a local representation of the relief at each point of the trunk mesh. Then, the automatic thresholding of Rosin [16] is used to segment the surface singularities. This method will be further detailed in Section 3.1.1. On ten meshes, the authors obtain an F1 score of 0.71. In 2020, they successfully classified the segmented regions [10] using a random forest learning process. The singularities regions were classified into four classes: branch, branch scar, bush, small singularities. The authors obtain an average of 0.86 F1 score on all classes, but only 0.46 on small singularities.

\subsection{Deep-learning Based Methods}

In a related context of the defect detection on surfaces of cylindrical objects, Tabernik et al. [17] proposed a method to detect surface defects on cylindrical metal parts. Grayscale intensity images of the surface are generated. A network of successive convolution layers and max pooling is used to segment cracks on the surface image. Then, a decision network made of a convolution layer and a final fully connected layer allows to decide whether or not there is a defect on the surface image. The proposed strategy is original and appears efficient for the specific case of defects on cylindrical metal parts. This is however not suitable to the singularities considered in our work.

The initial method [1] on which this paper relies can also be classified as a deep-learning based method. In particular, the learning process is performed on images generated from input meshes using the notion of delta distance [11]. The present strategy aims at improving the accuracy of the segmented regions. More precisely, the main idea of the method is to build a 2D view of tree bark surface from TLS data, called a relief map in order to train a CNN to the task of segmenting regions of particular intensities. The $2 \mathrm{D}$ segmentation is then redeployed back in $3 \mathrm{D}$ to extract the points corresponding to the singularity on the input mesh.

\section{Singularity Segmentation}

This section describes our method which consists of three steps (see Figure 3). Firstly, a 2D map is built from a 3D mesh of tree bark surface, called relief map (Section 3.1). This process stores the $3 \mathrm{D}$ point indexes in a $2 \mathrm{D}$ array, called discretization. In the second step, the singularities are 
segmented on the relief map by using a deep learning process allowing to overcome the complexity of the singularity shapes (Section 3.2). For this, we consider the U-Net architecture [14]. In the last step, the segmentation is used to recover the 3D points corresponding to the singularities thanks to the discretization (Section 3.2.2).

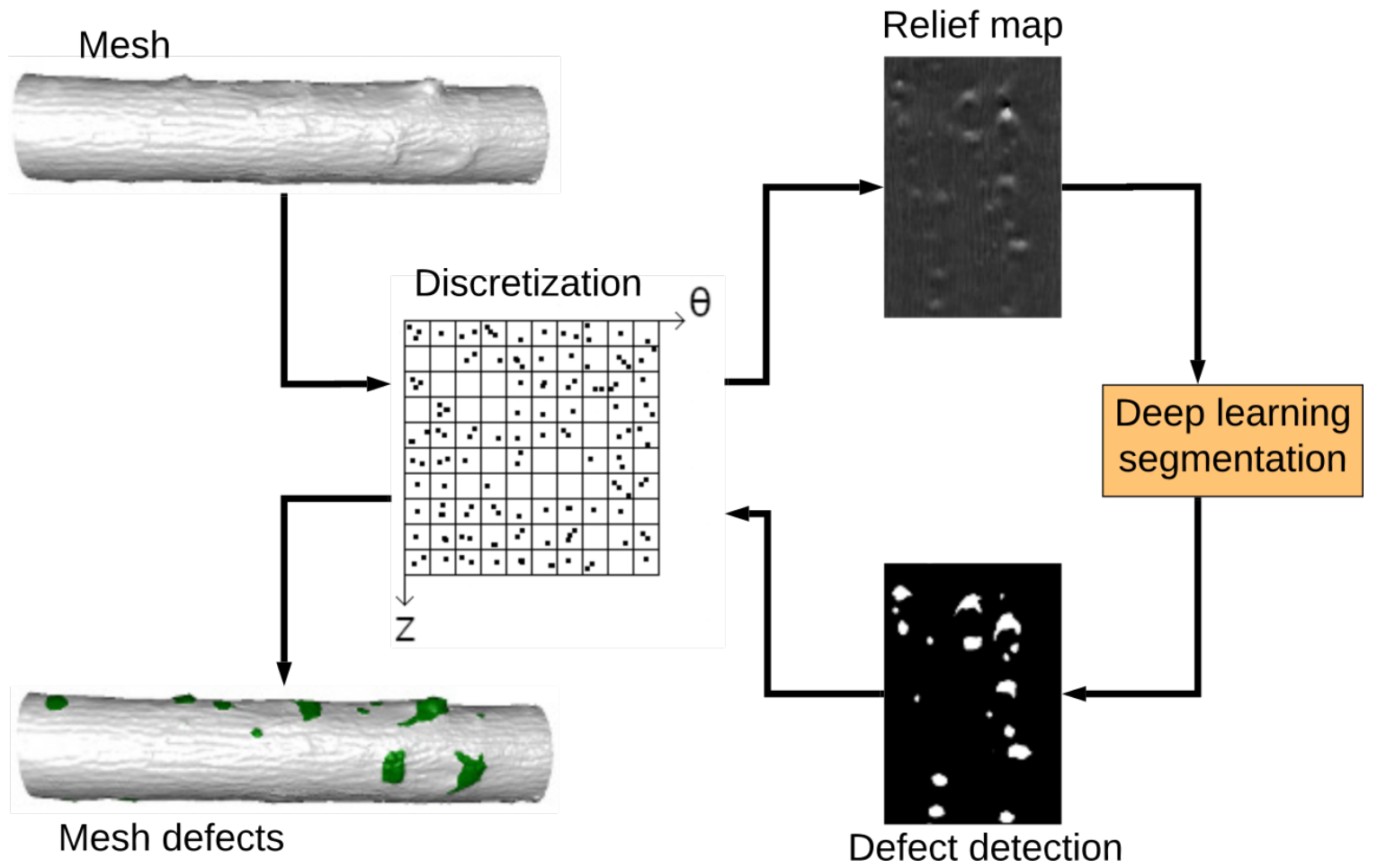

Figure 3: Pipeline of the proposed method [1].

\subsection{D Representation of 3D Mesh}

\subsubsection{Important Concepts from Previous Work}

We recall hereafter several concepts that are used in the rest of the article: (i) centerline extraction method [5, 6], (ii) cylindrical coordinate system, and (iii) local representation of the relief with the notion of delta distance [11, 12].

The centerline of a trunk consists of several segments. It is obtained by accumulating along the normals of the mesh faces in a voxel space and filtering with a confidence vote. As the trunk shape can be irregular, an optimization process is performed to obtain a smooth centerline (see Figure 4).

Due to the nature of the points on the trunk surface, we consider an alternative coordinate system, called cylindrical coordinates, which is simply a combination of the polar coordinates in the xy-plane with the $\mathrm{z}$ coordinate along the centerline. It allows to easily access neighbors of points on the trunk surface. More precisely, each point in Cartesian coordinate $P(x, y, z)$ is transformed into cylindrical coordinates $\left(r_{P}, \theta_{P}, z_{P}\right)$ with a local coordinate system $\left(C_{i}, u_{i}, v_{i}, w_{i}\right)$ defined for each segment $C_{i}$ of the centerline, such that

- $r_{P}$ is the distance between $P$ and $P^{\prime}$, the projection of $P$ on the segment $C_{i}$ of the centerline,

- $z_{P}$ is the height of $P$ along the centerline by summing the segment lengths of the centerline,

- $\theta_{P}$ is the angle between $P P^{\prime}$ and the axis $v_{i}$ of the local coordinate system associated to $C_{i}$.

The delta distance, noted by $\delta_{P}$, is a local representation of the tree bark relief. It is computed on a rectangular area $\mathcal{P}_{P}$ around the point $P$ (see Figure $5(\mathrm{a})$ ). The central straight line fitting the 


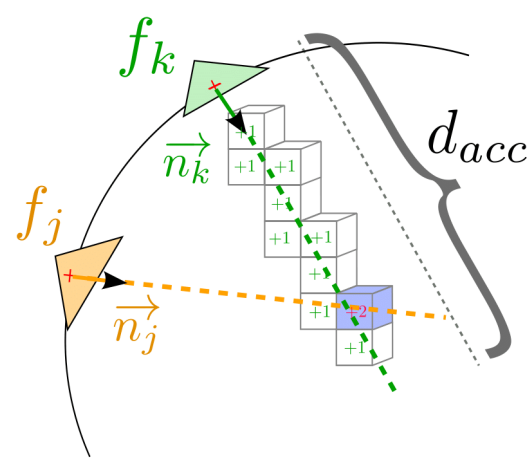

(a) accumulation process

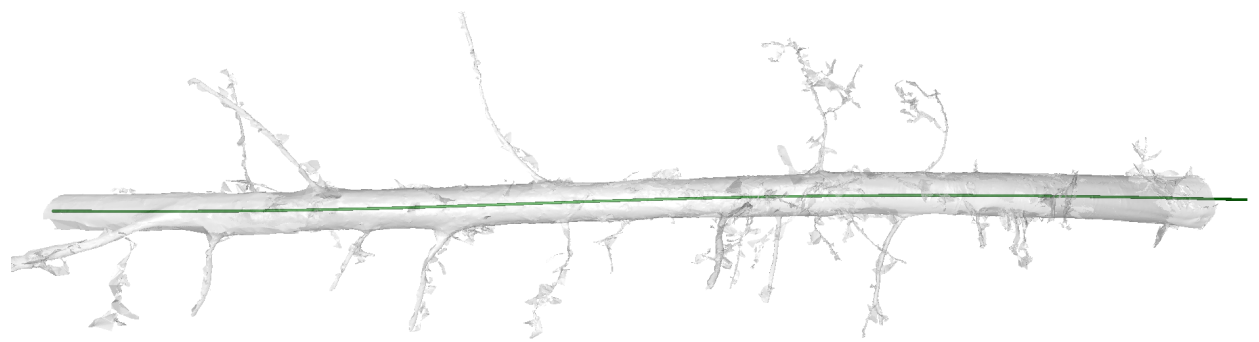

(b) centerline result

Figure 4: Illustration of the main idea of the centerline extraction algorithm: (a) accumulation step from surface faces $f_{k}$ and $f_{j}$ in the direction of their normal vectors $\left(\overrightarrow{n_{k}}\right.$ and $\left.\overrightarrow{n_{j}}\right) ;($ b) show the resulting centerline on the whole mesh.

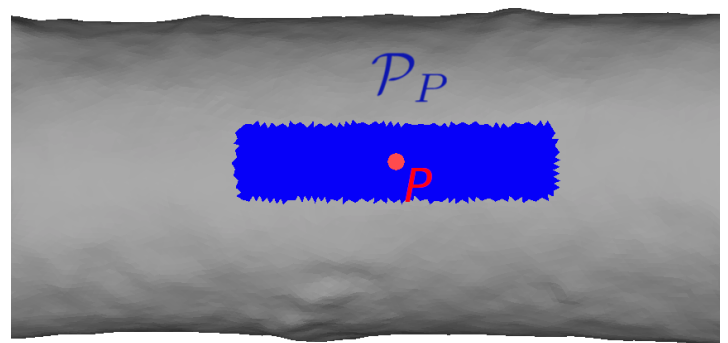

(a) Rectangular patch $\mathcal{P}_{P}$ centered on $P$

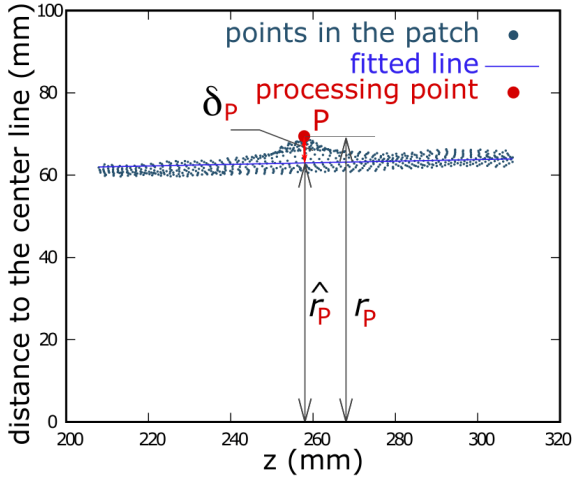

(b) Delta distance

Figure 5: (a) A patch in blue, associated to the red point $\mathrm{P}$, is used to compute the reference distance of this point. (b) Computation of $\delta_{P}$ for the red point. See [11] for more details.

points of $\mathcal{P}_{P}$ is calculated by a RANSAC based linear regression. The reference distance of the point $P$, noted by $\hat{r}_{P}$, is the distance from the projection of $P$ on this straight line to the corresponding segment of the centerline (see Figure $5(\mathrm{~b})$ ). The difference between $r_{P}$ and $\hat{r}_{P}$ represents the relief of the tree bark at the point $P: \delta_{P}=r_{P}-\hat{r}_{P}$. More details of this part can be found in [12].

\subsubsection{Discretization}

The points in cylindrical coordinates are used to construct a 2D array of height $\times$ width cells storing indexes of the corresponding points. This array is called discretizationMap. By convention, the first (horizontal) and second (vertical) dimensions are respectively the angle $\theta_{P}$ and height $z_{P}$ values. Thus, the discretizationMap's height is equal to the trunk height. It is computed as the difference between the $z$ components of the points having the greatest and smallest heights. The discretizationMap's width, as proposed in [1], is calculated as the average circumference of the trunk, forcing our segmentation method to work on complete trunks while the calculation of the centerline can work on partial trunks (in Figure 7 (a), the centerline is built on a mesh of a partial trunk). In this work, the discretization is improved by fitting the width of the discretizationMap with highest arc length of the mesh. To approximate this value, we consider a binary array $T$ initialized to 0 . The size of $T$ is set by the Pad parameter. This array is used to maintain an occupancy map and updated by going through all mesh points. For each point, its index $i$ on $T$ is computed from its $\theta_{P}$ angle of the 


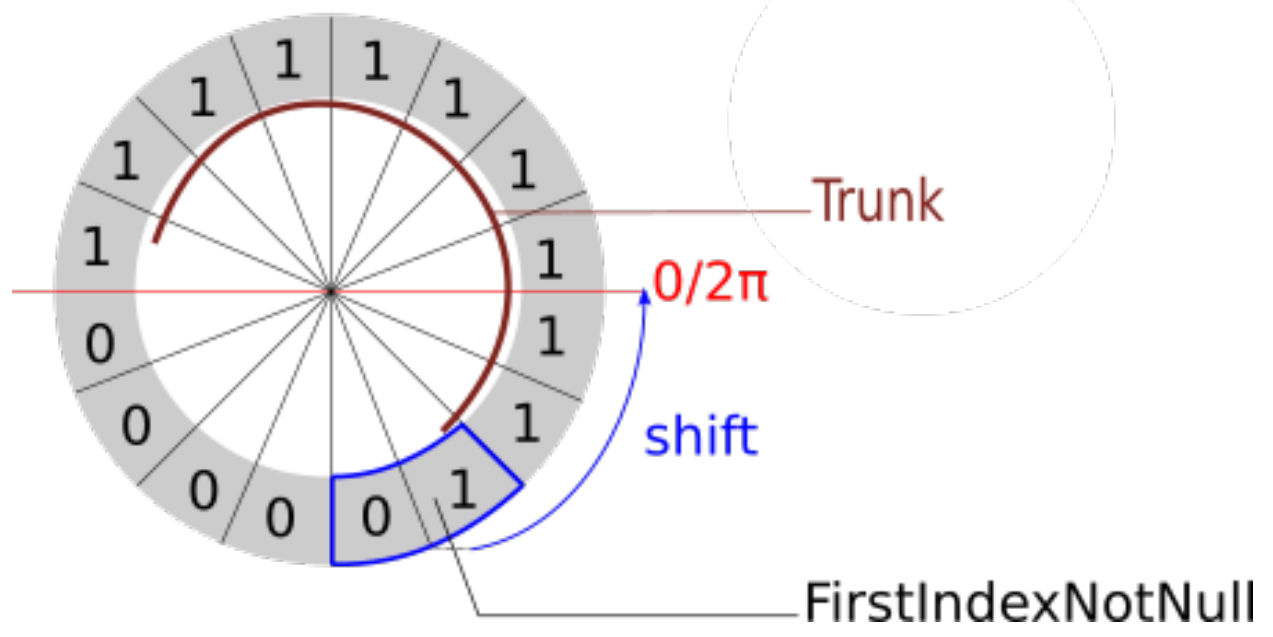

Figure 6: Computation of the partial circumference of the trunk.

cylindrical coordinate

$$
i=\left\lceil\theta_{P} * \frac{P a d-1}{2 \pi}\right\rfloor,
$$

where $\lceil x\rfloor$ represents the nearest integer to $x$. The array $T$ is marked at index $i$ of the point with value 1. Figure 6 shows an illustration of $T$ for $P a d=16$ where the mesh points have changed 11 array values from 0 to 1 . After all points have been processed, the arc length (discretizationMap's width) is computed as follows

$$
\text { width }=\left\lceil 2 \pi * \frac{(\text { Pad }-n b Z e r o)}{P a d} * \text { meanR }\right\rfloor,
$$

where $n b Z$ ero is the total number of 0 in $T$, and mean $R$ is the average radii $r_{P}$ of all points belonging to the trunk mesh. The width value is rounded to the nearest integer.

The discretizationMap has height $\times$ width cells. For each point of the mesh, we compute its position, pos $X$ and $\operatorname{pos} Y$, of the cell in the discretizationMap. The position pos $X$, associated with $\theta_{P}$, has to take into account the first value $f_{1}$ (the closest angle to $2 \pi$ or 0 ) which is associated with the angular interval computed from the binary array $T$. The trunk can be partial and therefore the angle interval can be reduced to $\left[f_{1}\right.$, upper $\left.B A-f_{1}\right]$ with upper $B A$ the angular coverage of the trunk. The value of upper $B A$ can be approximated using $T$

$$
\text { upper } B A=\left\lceil 2 \pi * \frac{(\text { Pad }-n b Z \text { ero })}{P a d}\right\rfloor \text {. }
$$

An angular shift is computed to replace the interval of $\theta_{P}$ between $[0$, upper $B A]$. This shift is obtained by looking for a pattern '01' (framed in blue in Figure 6) in T. The index, firstIndexNoNull, is the index at the position of the cell ' 1 ' of the pattern '01'. The shift is then given by

$$
\text { shift }=2 \pi-\left(\frac{\text { firstIndexNoNull }}{\operatorname{pad}} * 2 \pi\right) .
$$

Finally, pos $X$ and $p o s Y$ are given by

$$
\left\{\begin{aligned}
\operatorname{pos} X & =\frac{\text { width }-1}{\text { upper } B A} *\left(\left(\theta_{P}+\text { shift }\right) \bmod 2 \pi-\text { upper } B A\right)+\text { width }-1, \\
\operatorname{pos} Y & =z_{P}-\min H .
\end{aligned}\right.
$$


This step of discretization is described in Algorithm 1. In the code, it corresponds to the function computeDicretisation of the class UnrolledMap in the package ReliefMap.

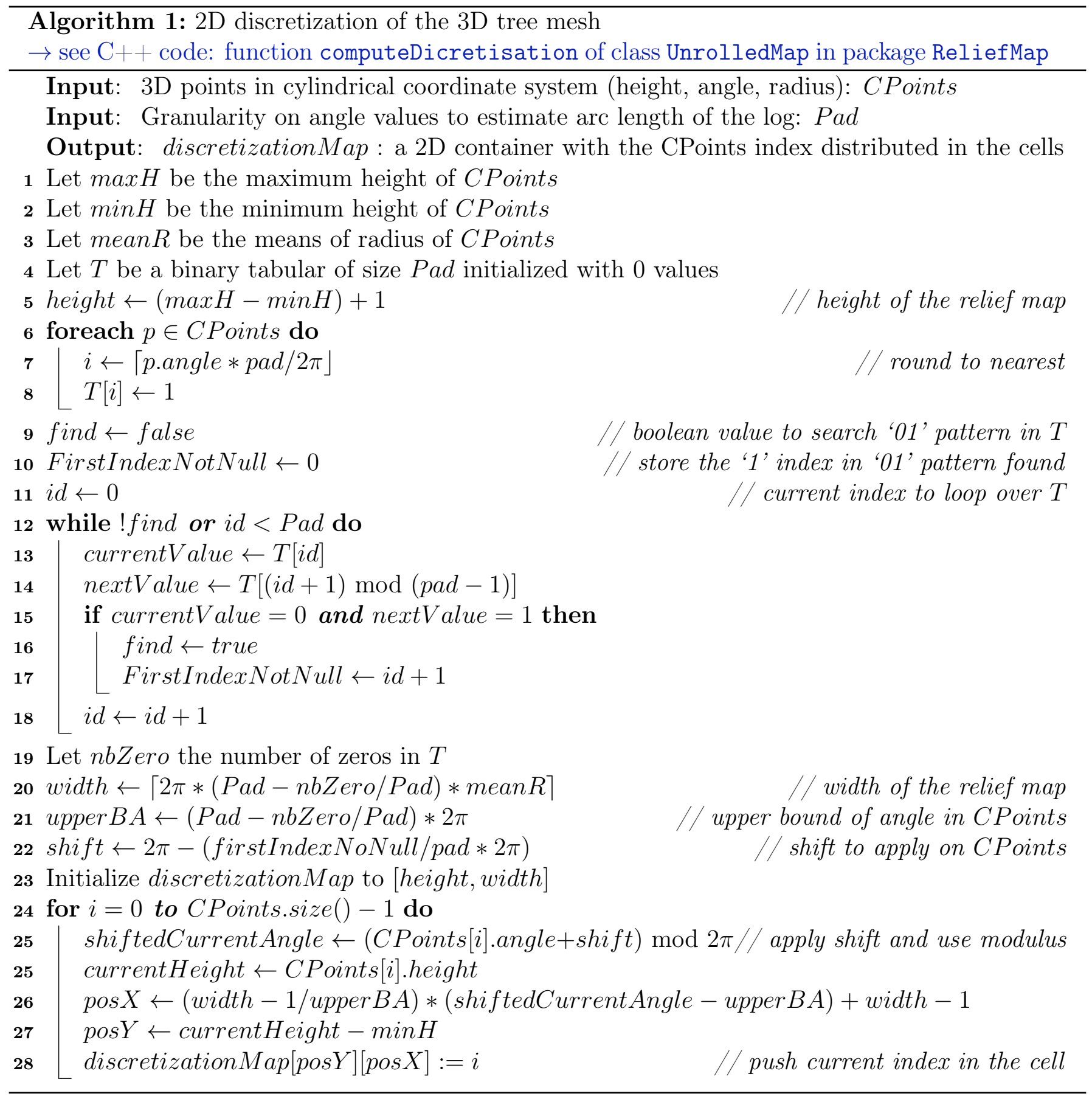

\subsubsection{Relief Map}

From the 2D structure - the discretizationMap - containing for each cell a set of index points, we will build an image in which the intensity of each pixel is calculated with the corresponding cells in the discretization. It is called a relief map. In the following, we describe the multi-resolution process (Algorithm 2) allowing to generate the graylevel relief map with fixed scale from the discretizationMap.

The relief map is built through the discretization process. The map domain is rectangular but the trunk does not necessarily cover the whole area. During the loop over the discretizationMap cells, 
we check if a pixel could be represented by an intensity. This consists in trying to reach the edges of the discretizationMap, if at least one cell is not empty during the process, then the pixel could be represented by an intensity. We see in Figure 7 (b), a relief map obtained from a partial trunk, the red pixels are those whose intensity will not be calculated during the discretization process. Algorithm 3 is used to check if a cell of the discretizationMap could be represented. The function corresponding to this algorithm is detectCellsIn in the class UnrolledMap of the package ReliefMap.

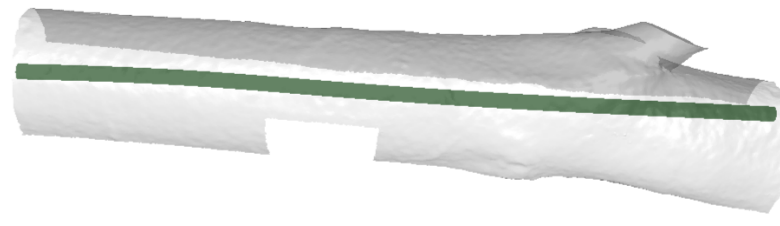

(a)

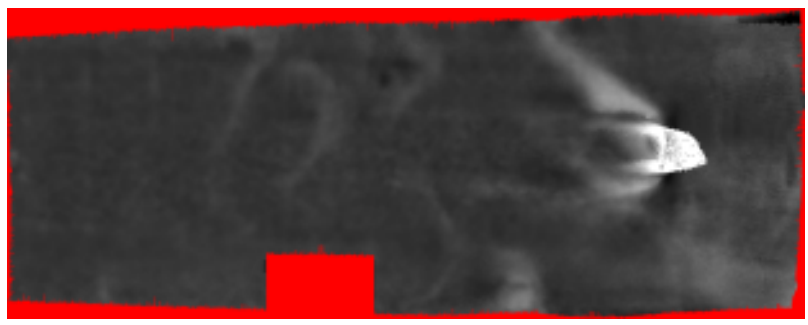

(b)

Figure 7: (a) Partial mesh with centerline in green. (b) Partial relief map with outside pixels in red.

For each cell, a value is chosen according to the delta distance $\left(\delta_{P}\right)$ of points in the cell. This distance $\delta_{P}$ represents the local relief of each point, its calculation is detailed in [11] and reported in Section 3.1.1. If the current cell is not empty in the discretizationMap, we choose the maximum $\delta_{P}$ of all points contained in the cell. Otherwise, a multi-resolution analysis is performed. It consists in looking for the missing information in cells resulting from the discretizationMap reduced by a factor $\frac{1}{2^{n}}$, with $n=1, \ldots, M a x d F$ until the cell contains at least one point (MaxdF is a parameter of the multi-resolution search function, it allows to limit the search). More precisely, the points contained in the larger cells are obtained by accumulating those of the unreduced cells of the discretizationMap on a square window of width $2^{n}$. Finally, we choose the maximum $\delta_{P}$ of points contained in the studied cell. Algorithm 5 allows to extract the points in lower resolution and Algorithm 4 gives the maximum relief representation of a set of points. They correspond respectively to the functions getIndPointsInLowerResolution and maxReliefRepresentation in the class UnrolledMap of the package ReliefMap.

A grayscale image is generated from relief map, by using a single static intensity scale where intensityPerCm grayscale levels correspond to one centimeter. Associated to this parameter, a cutoff interval of minimal and maximal heights $([\min R V, \max R V])$ is considered to ensure that all levels can be represented in the usual $[0,255]$ range. The minimal value $\min R V$ is a parameter of our method and the maximal value $\max R V$ is simply given by

$$
\max R V=255 * \frac{1}{\text { intensityPerCm }}+\min R V .
$$

The resulting grayscale value is deduced as follows

$$
\text { grayscaleValue }=\frac{\text { reliefValue }-\min R V}{\max R V-\min R V} * 255 .
$$

Algorithm 6 allows to generate the grayscale relief map. It is implemented in the function toGrayscaleImageFixed, in the UnrolledMap class of the package ReliefMap.

\subsection{D Segmentation}

The relief map allows a 2D representation of the trunk. The 3D problem of surface singularity detection becomes a 2D problem of binary-image-classification in which each pixel of the relief map 


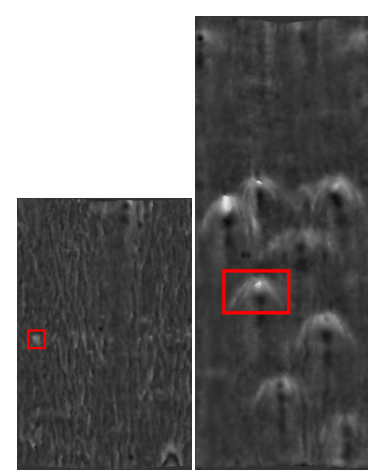

(a) (b)

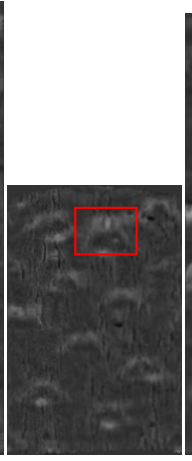

(c)

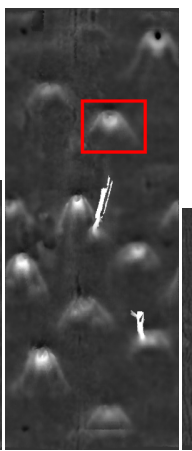

(d)

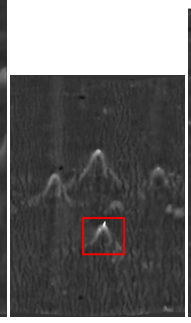

(e)

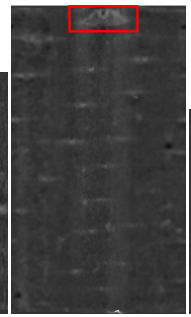

(f)

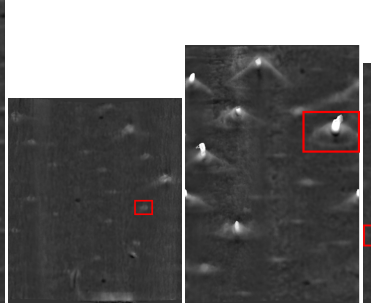

(g) (h)

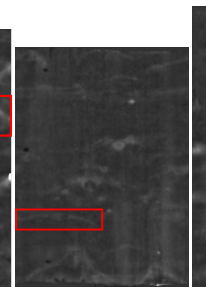

(i)

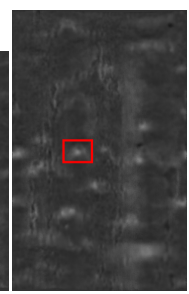

(j)

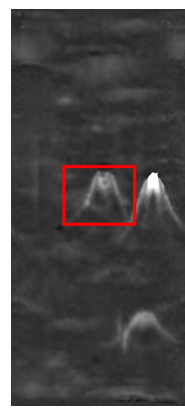

(k)

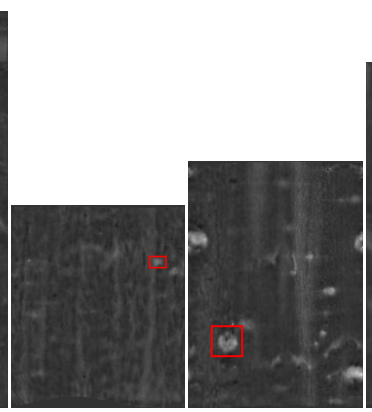

(1)

(m)

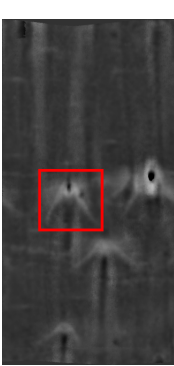

(n)

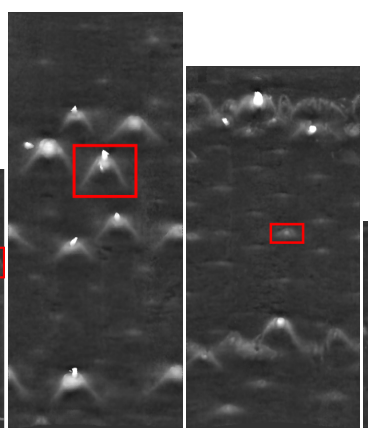

(p)

(q)

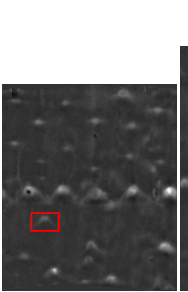

(r)

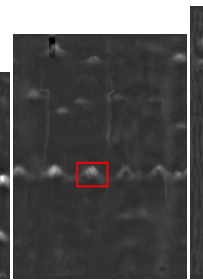

(s)

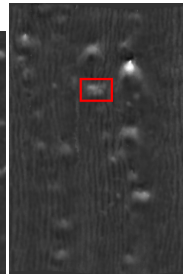

(t)

Figure 8: Examples of relief maps after multi resolution analysis. White color for stronger reliefs. Examples of singularities are highlighted in red frames, one singularity per relief map. Here is the correspondence between relief map and tree species: $(a, b, c, d)=$ Alder, $(e, f, g, h)=$ Aspen, $(i)=$ Beech, $(j, k)=$ Birch, $(i)=$ Horn beam, $(m)=$ Linden, $(n, o)=$ Red oak, $(p, q)=$ Wild cherry, $(r, s)=$ Fir, $(t)=$ Elm.

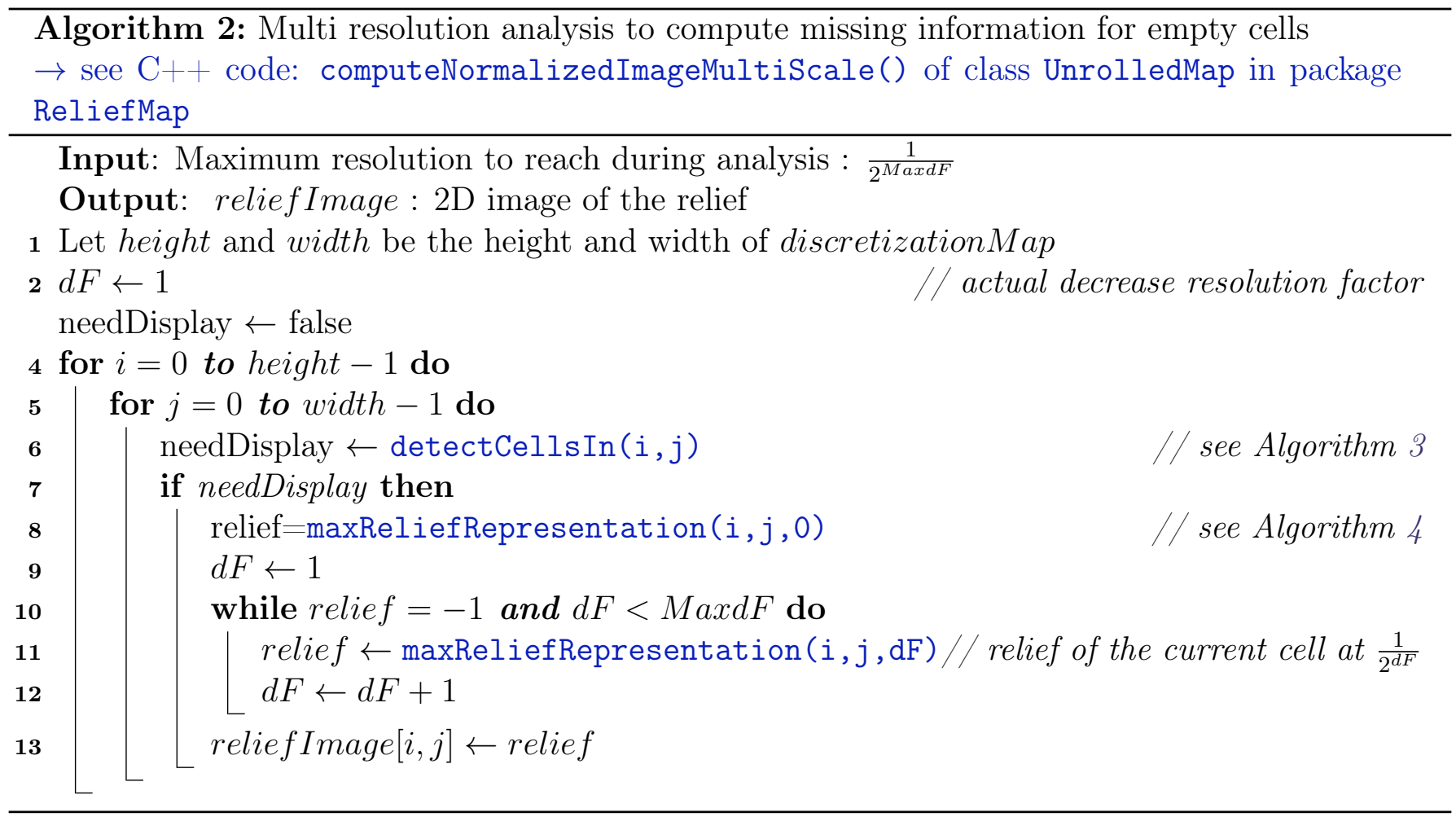




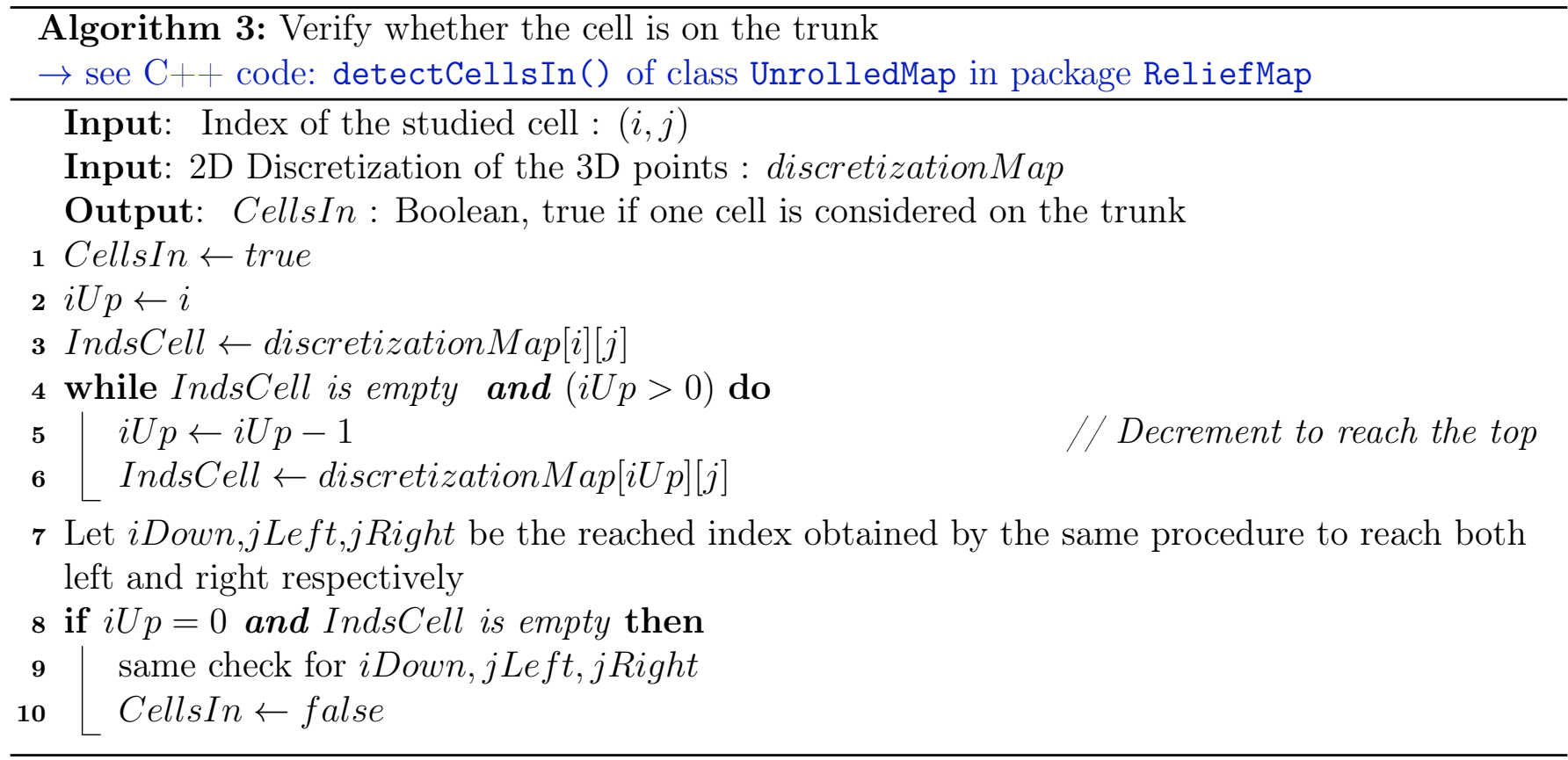

Algorithm 4: Compute relief representation at one cell in specified resolution

$\rightarrow$ see $\mathrm{C}++$ code: maxReliefRepresentation() of class UnrolledMap in package ReliefMap

Input: Index of the studied cell : $(i, j)$

Input: Decrease resolution factor : $d F$

Input: Relief representation for each 3D point: relief Representation

Output: maxRelief : relief representation of one cell with a specified resolution

IndexesInCell $\leftarrow$ getIndPointsInLowerResolution(i,j, dF) // see Algorithm 5

2 if IndexesInCell is not empty then

$3 \quad$ maxRelief $\leftarrow$ search for the maximum relief value in IndexesInCell else

$5\llcorner$ maxRelief $\leftarrow-1$ conditional value

Same procedure for to compute the mean or median relief representation

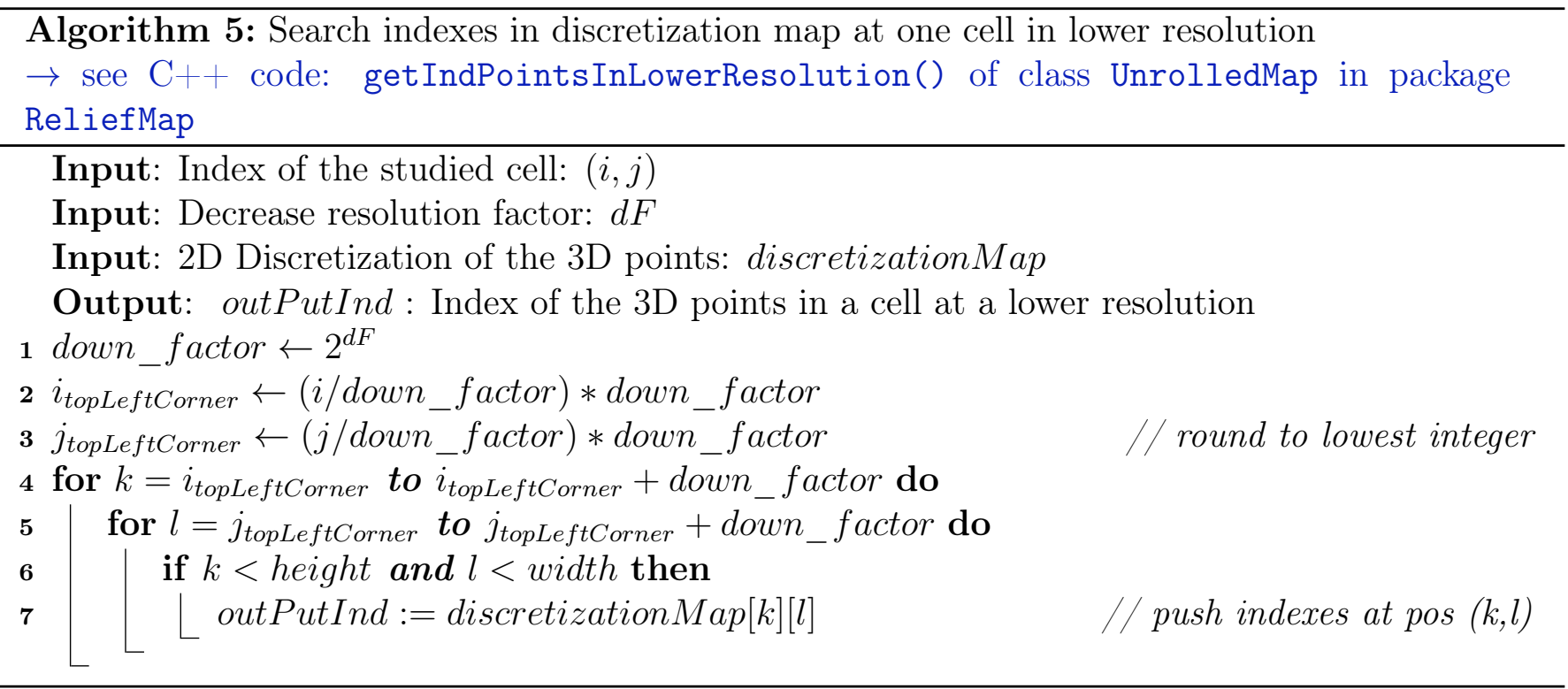




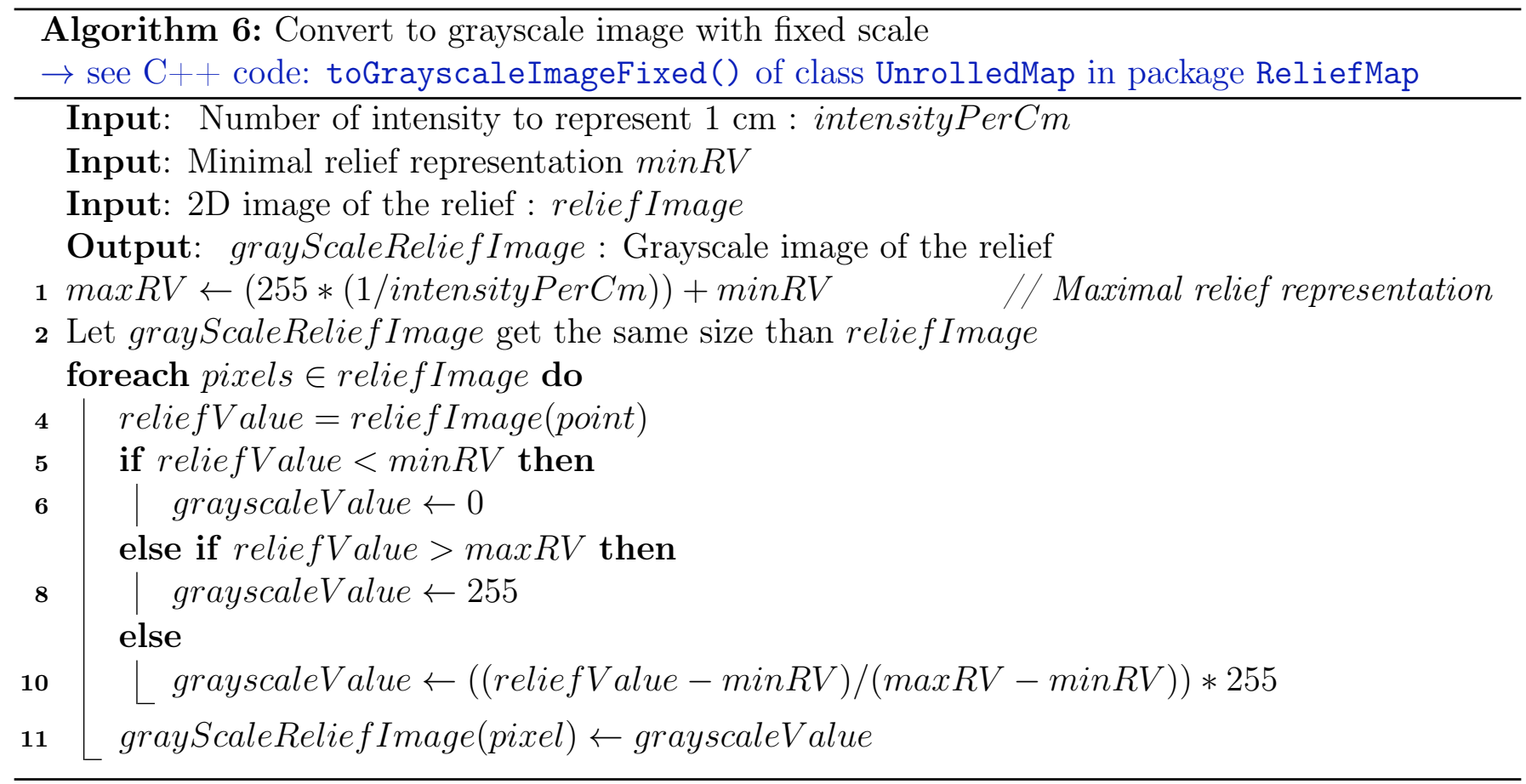

will be classified as singularity or not. We can see in Figure 8 that singularities on tree barks may have arbitrary size, shape and orientation. Furthermore, the roughness of the tree bark, the variability of singularities for the same species and between different species make the detection task difficult to automate by conventional segmentation algorithms. To overcome this, we opted for a segmentation by a supervised deep learning approach. In Section 3.2.1, we explain the generation of training pairs (example/label). Section 3.2.2 details the trained CNN architecture of U-Net [15].

\subsubsection{Training Data}

The singularities (or defects) have been hand-segmented on the meshes (and verified by experts). This information allows to associate to each relief map an image labeled in black and white. The passage of 3D points to 2D labeled image is done by a similar process as described in Section 3.1. The difference is that, instead of using $\delta_{P}$ to represent reliefs in gray level, the pixels corresponding to the singularities have intensity 255 (white) and the others have intensity 0 (black). Besides, as can be seen in Figure 9 (a), some pixels belonging to the singularities remain black. The multiresolution process is not used here because it would tend to enlarge the singularities. Instead, a closing and opening operation with a kernel of size $3 \times 3$ is applied on the labeled image (see Figure 9 (b)). In Figure 9 (c) we see the resulting relief map associated to the labeled image.

The size of the relief map (and the labeled images) is different from one mesh to another because the circumference and height of the trunk are not always the same. To increase the number of training data, we have extracted patches of size $320 \times 320$ pixels following two strategies:

1) with singularities: the process consists in finding the barycenters of connected components in the labeled images. For each barycenter, we extract a centered patch of size $320 \times 320$ pixels in both labeled image (as ground-truth) and relief map. If a singularity is too close to the edge of the image, a translation is performed so that the patch still contains the singularity and is included in the image (see Figure 10).

2) without singularities: the process consists in finding rectangular areas of size $320 \times 320$ pixels containing no white pixels in the labeled image. When such an area is found, the patch is 


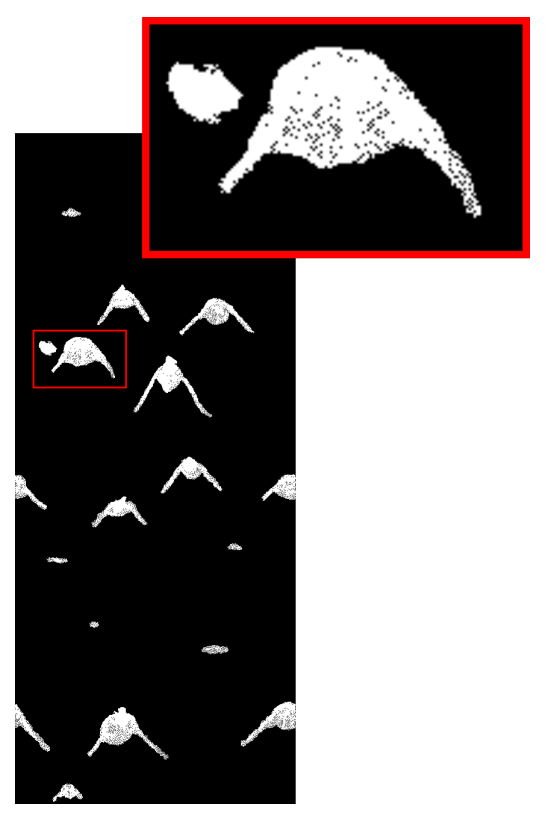

(a)

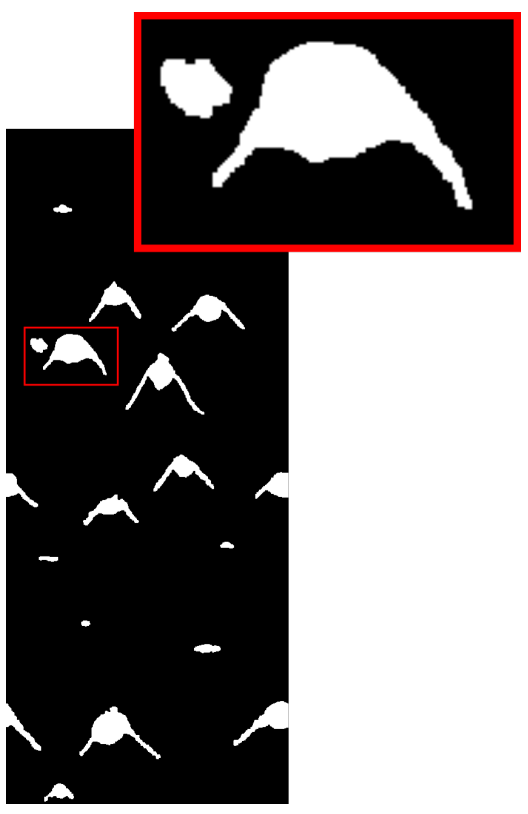

(b)

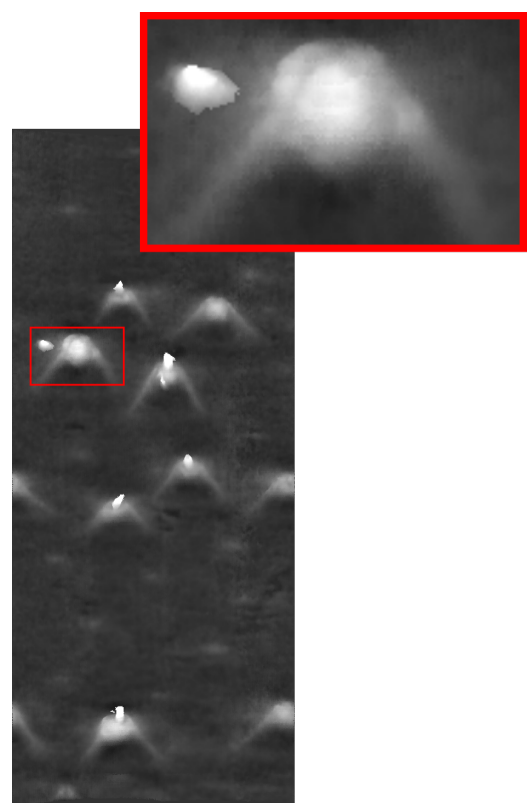

(c)

Figure 9: labeled image and corresponding relief map with zoom in rectangular area: (a) before closing and opening operation, (b) after closing and opening operation and (c) corresponding relief map.
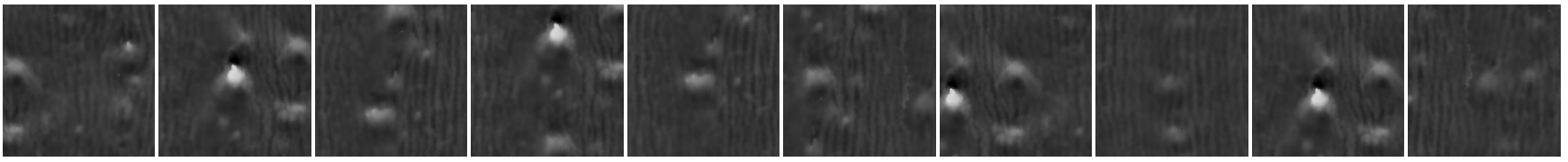

(a) Extracted patches with singularities from relief maps
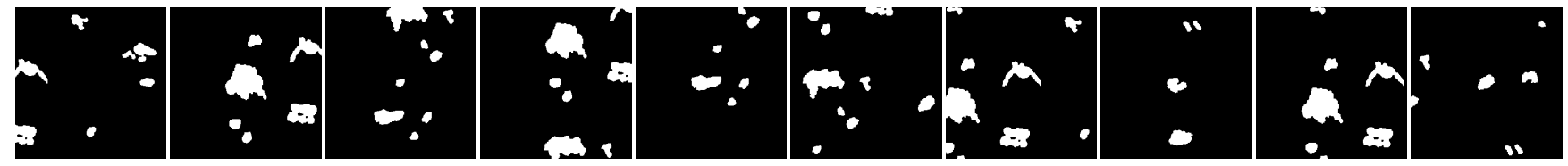

(b) Extracted patches with singularities from annotated maps

Figure 10: Some samples from training data.

extracted in the relief map and a black image is generated as ground-truth.

Following this splitting process, different techniques were also applied on the resulting patches to enlarge further the data. In particular, we consider the following operations: rotation in range $0^{\circ}$ to $20^{\circ}$, flipping vertically and horizontally, zooming of $\pm 30 \%$ and randomly deleting a rectangular area [2] with a 0.5 probability. Note that this data augmentation is performed on the fly, i.e., during the training process.

\subsubsection{U-Net Architecture for Segmentation}

U-Net is a fully convolutional network. It is widely used in bio-medical image segmentation [3, 19, 20]. More recently, U-net has been applied in other fields such as satellite image segmentation [4, 18] or tunnel object detection [13]. This architecture is well-known for its performance when trained with very few images.

In general, a U-net network consists of two parts: encoding and decoding. The encoding part looks like a classical convolutional network architecture. It consists in applying successively convolutions 
and max pooling operations. The decoding part is made of up-sampling operations followed by a concatenation with the layer of the same level in the encoding part.

For our problem of detecting tree bark surface singularities, we made several changes to the original U-Net. In order to reduce the over-fitting of the considered neural network, we apply a regularization technique, called drop-out. More precisely, two dropout layers are added, with a 0.5 probability, to randomly drop some of the connections between layers. At the first level, there are 32 filters per convolution layer. Then the number of filters is doubled at each level. Besides, due to the dying ReLU problem [9] the Leaky ReLu activation function is employed instead of ReLU from the original architecture. Finally, in the last layer, we use a sigmoid activation function instead of the soft-max function to ensure the output pixel values range between 0 and 1 . The proposed network architecture is illustrated in Figure 11.

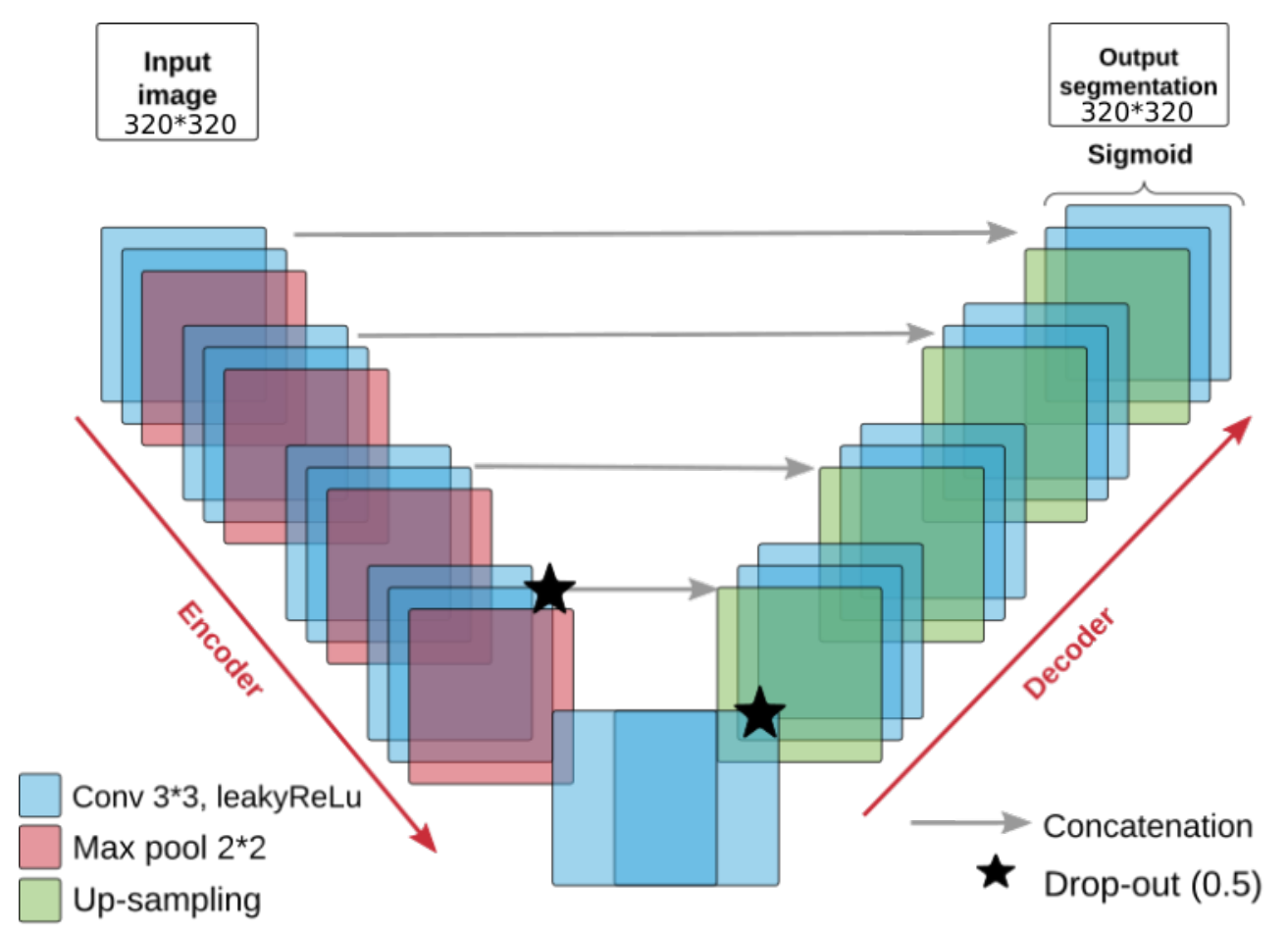

Figure 11: The architecture for tree bark singularities detection based on U-Net [15].

Once trained, the network's prediction produces an image whose pixel intensity corresponds to a probability score of belonging to a singularity pixel. The higher the grey level, the more the pixel is considered as a singularity by the network and vice versa. We can see in Figure 12 two examples of output of the trained network.

From the prediction result, a threshold is applied to retrieve a 2D segmented image in black and white. The white pixels correspond to the regions of the relief map considered as singularities. In order to obtain the singularities on 3D mesh from white pixels of the segmentation results, an inverse discretization is performed. More precisely, the discretization has the same size as the segmentation. The indices of the points contained in the cells of the discretization at the position of the white pixels are extracted. They correspond to the singularities in 3D. In Figure 12, we can see two examples of extracted points in green color from the segmented results. 

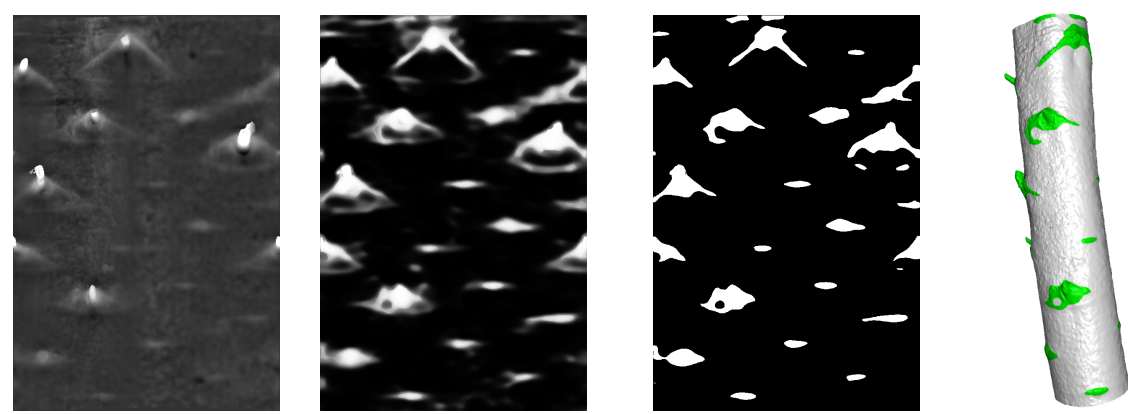

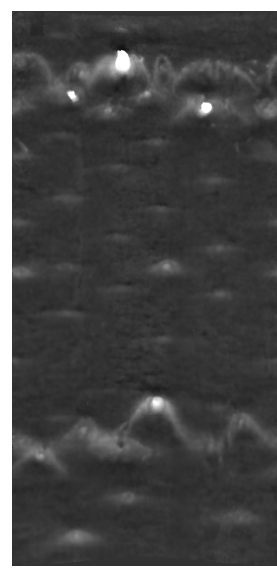

input

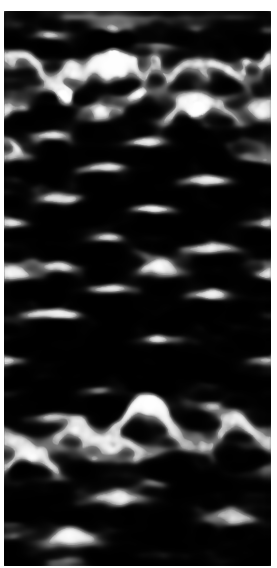

output

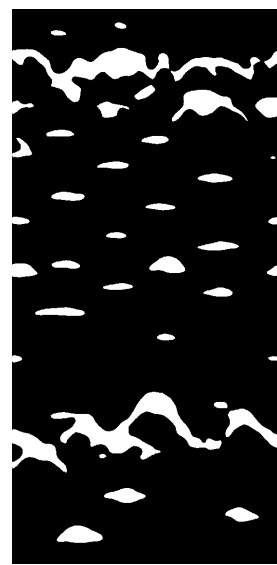

binary output $(\mathrm{t}=0.5)$

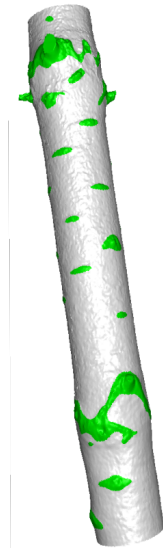

mesh

Figure 12: Examples of output of our U-net [15] inspired architecture.

\section{Experiments}

\subsection{Dataset}

To test our method we have 25 trunk meshes: 10 from [11] and 15 new data manually annotated. This database contains 11 tree species: beech, birch, elm, fir, red oak, wild cherry, aspen, horn beam, lime, and alder. The studied trunks have between 366 and $896 \mathrm{~cm}$ of circumference and between 694 and $1150 \mathrm{~cm}$ of height. The visible surface singularities are mainly branch scars and burls, there are also some small singularities whose type is not distinguishable. In Figure 13, we can see some examples of 3D point clouds from the dataset. We randomly pick 5 trunks among the 25 to test the method and kept the others for training.

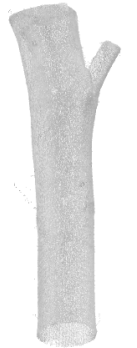

Beech

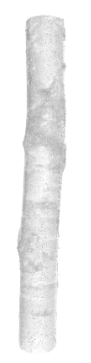

Birch

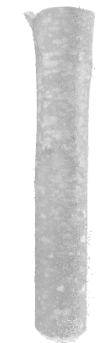

Red oak

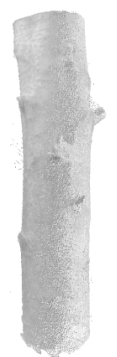

Aspen

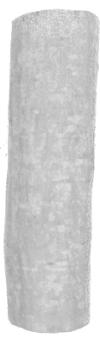

Charme

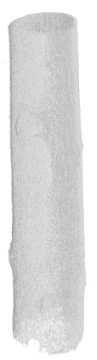

Linden

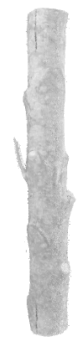

Alder

Figure 13: Examples of trunks (logs) of different tree species, captured from LiDAR point cloud including reflectance values. 


\subsection{Cross-validation Studies}

The cross-validation consists in training $k$ models by varying the data used for training and validation in such way that the model is validated on $k$ different folds. In our experiment $k=5$. The relief maps, generated from the training data, are split into patches of $320 \times 320$ pixels (see Section 3.2). After the splitting process, we obtain 456 patches. Five models are trained on groups of patches with a ratio of $\frac{1}{5}$ for the validation and the rest for the training. These groups of patches are constructed so that the validation data is different for each model. Once the network is trained, we apply the segmentation method (see Section 3) on the 5 test trunks, and obtain a set of points classified as singularities. They are then compared to the ground-truth. It should be mentioned that, in [1], we performed a cross validation on the same data. However, the experiment was different, we wanted to test our method on all the available data. Here, the cross validation allows us to test the performances of the segmentation model and its sensibility to the learning data. Figure 14 summarizes the difference between the two cross validation procedures on a base of ten examples.

The training process was performed on a GPU (NVIDIA Geforce RTX 2080Ti with 12Go RAM). We used the Adam optimiser [7] with the two parameters $\beta_{1}=0.9$ and $\beta_{2}=0.99$ (default values in tensorflow), and set the learning rate at 0.0001 . We trained our network for 40 epochs, each epoch comprised 111 steps with 10 images per batch. As for the parameters dropout rate $\delta$ and Leaky ReLu activation $\alpha$, several values have been tested, and we come out with $\delta=0.5$ and $\alpha=0.01$ for the smallest loss function (binary cross entropy) on the validation set. It should be mentioned that our training is quite fast, it takes about 14 seconds per epoch.

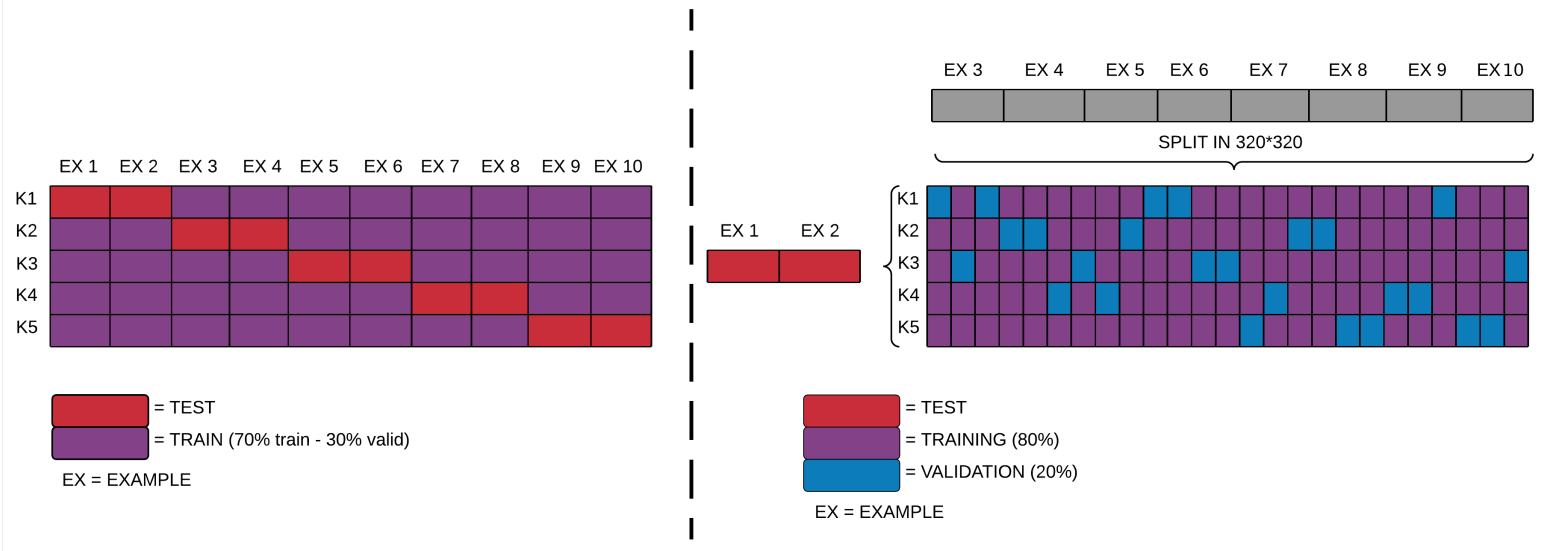

Figure 14: Difference between the cross validation in [1] (left) and our cross validation (right).

\subsection{Results}

The results are presented in Table 1. For evaluating the method, 3 metrics are used: precision, recall and F1 score. Each row contains the results of the training model on the test data. The average and standard variation are calculated over the 5 models. Figure 15 shows the segmentation results of our method (using $K 4$ as model segmentation) with a comparison to the ground-truth. On the examples WildCherry1, Redoak1 and Fir1, we obtain an average F1 score $>0.815$. The F1 score is lower on Aspen2 and Alder2. For Aspen2, we notice a decrease of the recall value compared to the other examples. The regions segmented by our method correspond well to singularities, however the ground-truth covers wider zones of defects. This results in an increase of the false negative rate and a decrease of the recall value. For Alder2, it is the precision value that is lower. Around the branch scars, the trunk forms cavities. Our segmentation method tends to encompass these 
structures which increases the false positive rate and therefore the accuracy decreases. Despite this difference in performance, the low values of standard variation show that the 5 models provide close performance measures even for Aspen2 and Alder2. This makes us believe that the training is robust to the considered dataset.

\begin{tabular}{|c|c|c|c|c|c|c|c|c|c|c|c|c|c|c|c|}
\hline \multirow{2}{*}{ K-Folds } & \multicolumn{3}{|c|}{ WildCherry1 } & \multicolumn{3}{|c|}{ Redoak1 } & \multicolumn{3}{|c|}{ Fir1 } & \multicolumn{3}{|c|}{ Aspen2 } & \multicolumn{3}{|c|}{ Alder2 } \\
\hline & prec & recall & F1 & rec & recall & F1 & prec & recall & F1 & rec & recall & F1 & prec & recall & F1 \\
\hline K0 & 0.825 & 0.88 & 0.851 & 0.893 & 0.886 & 0.889 & 0.831 & 0.815 & 0.822 & 0.877 & 0.561 & 0.682 & 0.594 & 0.878 & 0.707 \\
\hline K1 & 0.837 & 0.829 & 0.831 & 0.867 & 0.861 & 0.863 & 0.867 & 0.731 & 0.79 & 0.816 & 0.545 & 0.652 & 0.567 & 0.799 & 0.663 \\
\hline K2 & 0.831 & 0.868 & 0.848 & 0.878 & 0.877 & 0.877 & 0.857 & 0.778 & 0.814 & 0.894 & 0.611 & 0.725 & 0.593 & 0.831 & 0.691 \\
\hline K3 & 0.846 & 0.862 & 0.853 & 0.853 & 0.889 & 0.87 & 0.854 & 0.802 & 0.826 & 0.849 & 0.609 & 0.709 & 0.6 & 0.86 & 0.706 \\
\hline K4 & 0.811 & 0.911 & 0.857 & 0.871 & 0.906 & 0.888 & 0.827 & 0.82 & 0.823 & 0.88 & 0.636 & 0.737 & 0.596 & 0.885 & 0.711 \\
\hline Mea & 0.83 & 0.87 & "0.848 & 0.872 & $\overline{84}$ & 0.877 & 847 & "0.789 & 0.815 & $\overline{633}$ & "0.592 & 0.701 & 0.59 & "0.851 & 0.696 \\
\hline Std & 0.013 & 0.03 & 0.01 & 0.015 & 0.016 & 0.011 & 0.017 & 0.036 & 0.014 & 0.031 & 0.038 & 0.034 & 0.013 & 0.036 & 0.020 \\
\hline
\end{tabular}

Table 1: Cross validation results for the considered 5 folds.

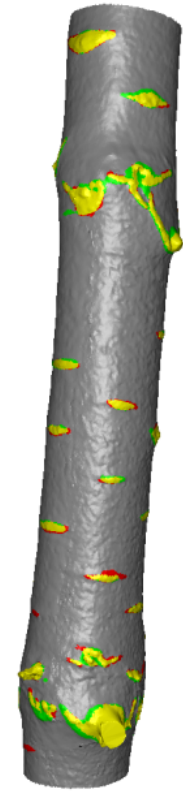

WildCherry1

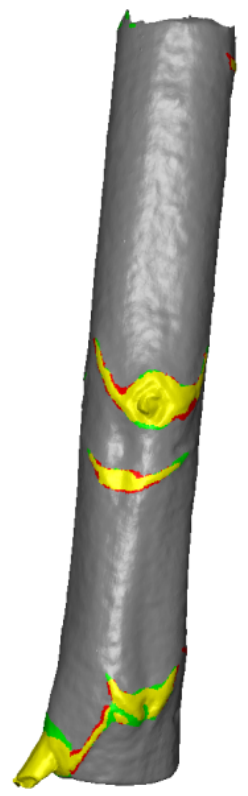

Redoak1

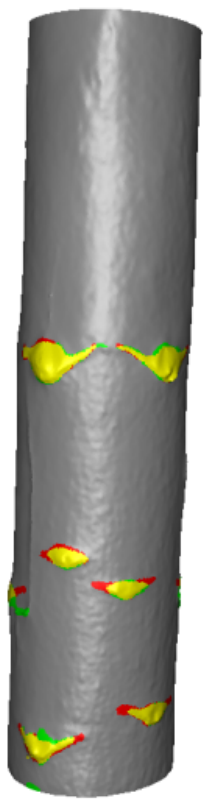

Fir1

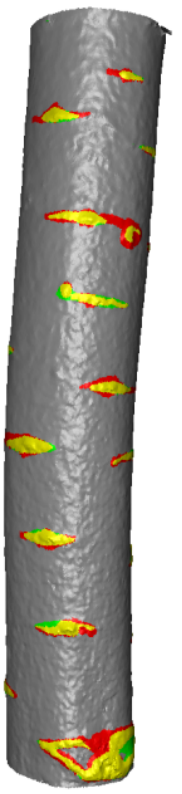

Aspen2

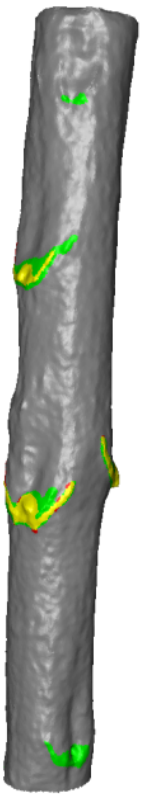

Alder2

Figure 15: Colorized mesh to show the difference between the ground-truth and segmentation obtained with the proposed method. Yellow is true positive, red is false negative and green is false positive.

\section{Code}

The code is written in $\mathrm{C}++$ and python. The $\mathrm{C}++$ part allows the interaction between mesh and relief map. The python part allows to use the segmentation model. In addition, the user can reproduce the results in Table 1.

\subsection{Materials}

The archive examples_IPOL.tar.gz contains the tree meshes and the ground-truth data files. They are in two directories: INRAE1a, INRAE1b. The data come from the same acquisition device and have 
been transformed into a 3D mesh using the process described in [10]. Each tree trunk is associated to three files: the mesh (with extension .off), the point indices corresponding to singularities (suffixed by -groundtruth-points.id), the face indices corresponding to singularities (suffixed by -groundtruth.id). The experiments in Section 4 use the files suffixed by-groundtruth.id for evaluating the performance of our method. The scaledMeshes directory contains scaled meshes used in Section 6.4.

The archive model_IPOL.tar.gz contains the models to reproduce the results. They are suffixed by $\mathrm{K}$ following a number between 0 and 4 . Images in Section 4 are generated from the model k4.hdf5.

\subsection{Dependencies}

For compilation of the $\mathrm{C}++$ sources these libraries must be installed:

- DGtal 1_1_0 or later: https://github.com/DGtal-team/DGtal

- GNU GSL: https://www.gnu.org/software/gsl/

- PCL: https://pointclouds.org/downloads/

To use our trained model for the segmentation of singularities on the relief map these libraries need to be installed (we recommend the user to use a virtual environnement as described in the tensorflow guide):

- Tensorflow 2.2: https://www.tensorflow.org/install/pip

- tensorflow-addons: https://www.tensorflow.org/addons/overview

- OpenCv: https://pypi.org/project/opencv-python/

\subsection{Generate the Relief Map $(\mathrm{C}++)$}

Once the dependencies are installed and the sources downloaded, the project is built with the following commands:

\section{cd TLDDC}

mkdir build

cd build

cmake .. -DDGtal_DIR=/path/to/DGtalSourceBuild

make

Three executable files are generated in the build directory: segunroll, groundtruth, segToMesh. segunroll allows the user to build the grayscale relief map. The image format is pgm. Three files are generated: reliefmap_info, cylPoint, discretize. The file reliefmap_info contains data relative to the construction of the relief map: height, width, upper bound of the angle of the mesh points (see Section 3.1.2). All the points of the mesh converted into cylindrical coordinates are stored in cylPoint using one point per line with: radius, angle and height. The file discretize contains height $\times$ width lines. Each line contains the list of indices of points belonging to the cell of the discretization (-1 if none). We save this information to avoid having to recalculate it later. The user can use the following command to generate the relief map:

./segunroll -i InputMesh [-h] [CenterlineParameters] [ReliefMapParameters] -o outputName

- InputMesh: path to a trunk mesh. 
- $-h$ : option for the command line helper.

- CenterlineParameters: chosen according the recommended values in [10].

- ReliefMapParameters: parameters for relief map (--MaxdF, --minRV,--intensityPerCm, --pad). They are also set with default values.

- $-o$ : prefix of the output.

For example, you can use this command to reproduce the same relief map used in our experiments:

./segunroll -i INRAE1a/WildCherry2.off -o WC2 -MaxdF 4 -minRV -5 -intensityPerCm 10 -pad 200

groundtruth allows the user to generate a pgm image of the same size as the relief map in black and white with white for the position of defects on the map. For this, the information previously calculated and stored in reliefmap_info and cylPoint is used. These labeled images are used to train our segmentation model, the user can generate them by using the following command (after generating the files reliefmap_info and cylPoint):

./groundtruth -i InputGrouthTruth -o outputName

- InputGrouthTruth: path to ground-truth, suffixed by: -groundtruth.id

- - : prefix the output.

For example, you can use this command to reproduce the ground-truth image used in our experiments:

./segunroll -i INRAE1a/WildCherry2-groundtruth.id -o WC_GT

segToMesh allows to extract the 3D points from the output of the segmentation model. The file discretize allows to make the correspondence between the white pixels (for singularities) and the 3D points of the mesh (see Section 3.2.2). Two files are generated. A file suffixed by -defect.id contains the indices of points considered as singularities. A file suffixed by defect.off contains the trunk mesh with the singularities colored in green. The command to do this operation is the following (after generating the singularity segmentation by an already trained model):

./segToMesh -i InputMesh -s segmentationMapName -o outputName

- InputMesh: path to the same mesh used to generate the reliefmap.

- $-s$ : path to the segmentation file.

- $-o$ : prefix the output.

The interaction between these different executables is summarized in Figure 16.

To generate the relief map and labeled images of a whole directory at once, you can use the script makeAllPair.sh (in the run directory) with the following command. The relief map will have the same name as the mesh and the labeled image will be fixed by _GT.

./makeAllPair.sh PathToMeshDirectory OutputPath

- PathToMeshDirectory: path to the input directory containing meshes.

- OutputPath: path to output directory in which all samples will be moved. 


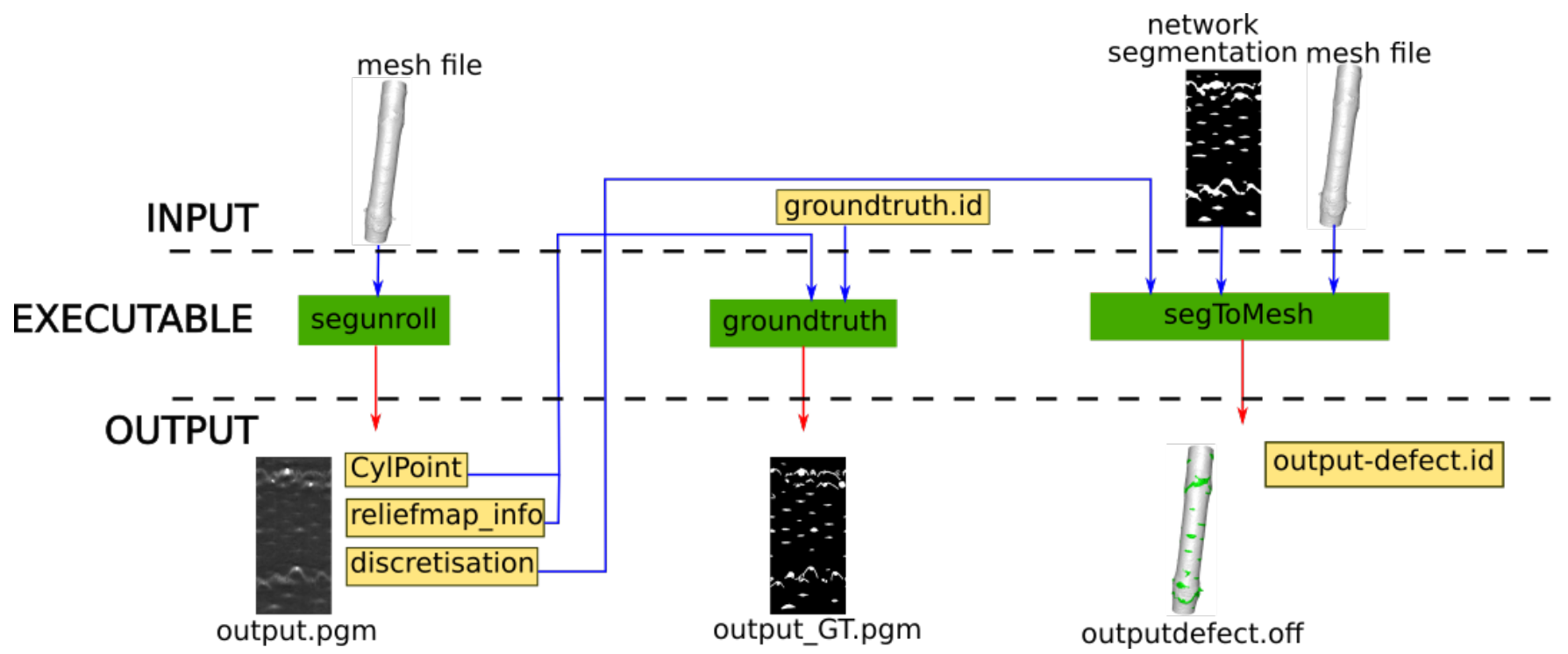

Figure 16: Interaction between the different executables of the project.

\subsection{Extract Patches from ReliefMap and Ground-truth Image (python)}

In the run directory, there is a script named train-valid_cut.py. From a relief map and a groundtruth image, it allows to extract training patches (see Section 3.2.1) and distribute them in the train and valid directories. From the created patches $80 \%$ are distributed in the train directory, and $20 \%$ in the valid directory. These directories must be created before calling the script and both of them must contain a directory named input and another named output. Here is the command to run:

python3 train-valid_cut.py -i PathToReliefMap -g PathToGT -o outputDir

- PathToReliefMap: path to input relief map.

- PathToGT: path to input ground-truth image.

- outputDir: output directory that must contain two directories named train and valid, which must contain two directories named input and output.

To perform the patches extraction on all pairs of training images in a directory (generated by the script makeAllPair.sh) you can use the script named train-valid_cut_All.sh. Here is the command to run:

/train-valid_cut_All.sh PathToInputDir PathToOutputDir

- PathToInputDir: path to input directory that contains relief map and corresponding groundtruth image suffixed by_GT.

- PathToOutputDir: output directory that must contain two directories named train and valid, which must contain two directories named input and output.

\subsection{Use our Trained Network (python)}

In the run directory, there is a script named predict.py that allows to use the network to segment the singularities on a relief map. It takes as input an already trained model and a relief map (previously generated) in pgm format. Two images suffixed by SEG.pgm and SEGTRESH.pgm are generated. The first one is in grayscale, the second one is in black and white (see Section 3.2.2). Here is the command to run: 
python3 predict.py InputReliefMap PathToModel Threshold

- InputReliefMap: path to the relief map.

- PathToModel: path to the model.

- Threshold: threshold $([0 ; 255])$ to apply on the network prediction.

\subsection{Train a Network with ReliefMap Samples (mix)}

Relief map and ground-truth images must be generated by the script makeAllPair.sh. Here is an example of how to generate these images from the INRAE1b directory and move them to the $\mathrm{db}$ directory:

./makeAllPair.sh examples/INRAE1b/ db/

The learning process requires data to be organized in a directory named train and another valid. In both of them, there must be a directory named input and another named output. Create them in a directory named $\mathrm{db}$. The network is trained on patches of size $320 \times 320$ (see Section 3.2.1), use train-valid_cut_All.sh to extract these patches:

.$/$ train-valid_cut_All.sh $d b / d b /$

Once data is prepared, train the network with the script named train.py. Here is the command:

python3 train.py -i $d b /$-o prefix

- $d b /:$ database directory that contain train and valid sample.

- prefix: name of the model.

\subsection{Test the Complete Process of our Segmentation Method (mix)}

In the run directory, there is a script named deep-segmentation.sh. This script allows to call in succession: segunroll, predict.py, segToMesh. Here is the command to execute it:

\section{./deep-segmentation.sh PathToModel PathToMesh}

- PathToModel: path to the model.

- PathToMesh: path to the mesh.

\subsection{Reproduce the Results (mix)}

In the run directory, there is a script named testK_folds.sh. This script allows to reproduce the results in Table 1. The segmentation method is tested on 5 examples and 5 models. The name of the tested mesh is written in the file testKFold_Path.txt at the root of the code repository. The scripts segunroll, predict.py, segToMesh are called to extract the singularity points of the mesh. The metrics (precision, recall, F1) are displayed, for each fold, in the terminal. Here is the command to execute it: 
- PathToMeshDirectory: path to the root of downloaded mesh directory.

- PathToModeleDirectory: path to the root of model directory.

\section{Influence of Parameters and Limits}

The relief map is the main tool of our method. It greatly influences the quality of the singularity segmentation. To generate a relief map, there are 4 important parameters. In the following, we describe the role and the default values of these parameters.

\subsection{Depth Parameter in Multi-resolution Analysis: MaxdF}

A multi-resolution analysis is performed on the relief map. The purpose of this analysis is to complete the relief map with information at lower resolution (see Section 3.1.3). During this operation, the dimensions of the relief map are reduced by a factor of $\frac{1}{2^{n}}$ with $n \in[0, \ldots, M a x d F]$. In Figure 17, we can see 5 relief maps coming from the same mesh at different resolutions which decrease by a factor of two. At the resolution 1, we can see "holes" (pixels with no value) on the relief map, this missing information will be completed by analyzing the relief map at a smaller resolution. By reducing the resolution of the relief map, the information it contains is reduced as well (the relief is less well represented). We stop the multi-resolution search, when the resolution becomes too small. The MaxdF parameter allows us to stop this search. If $n=\operatorname{MaxdF}$, the multi-resolution process is stopped at the first level. In our data, the smallest resolution reached by the multi-resolution analysis is $\frac{1}{2^{4}}$. Therefore, we set $M a x d F=4$ as the default value.
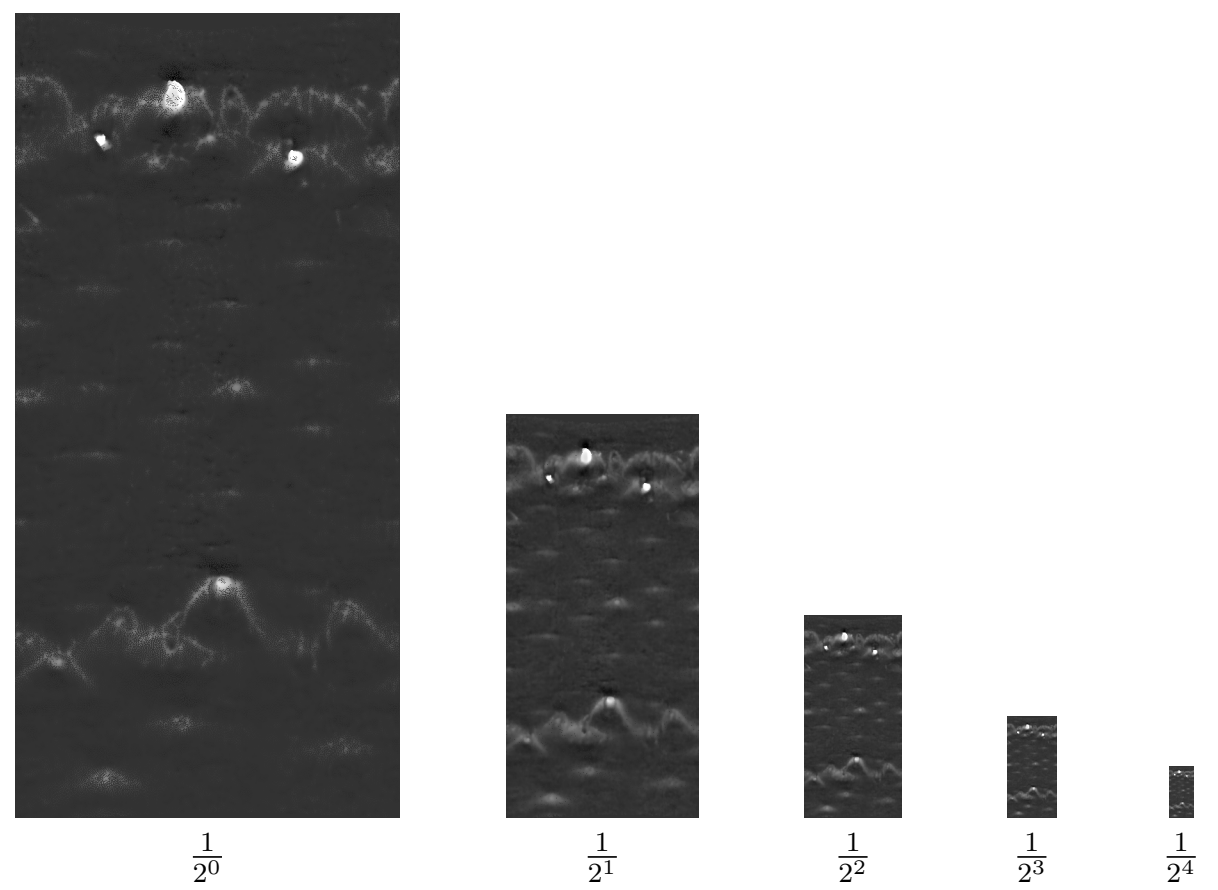

Figure 17: Same relief map with resolution decreasing by $\frac{1}{2^{n}}$ factor with $n \in[0, \ldots, 4]$. 


\subsection{Grayscale Parameters: intensity_per_cm and minRV}

The singularities are distinguishable from the rest of the trunk because they form a relief variation on the surface. This variation can be negative or positive. To represent the surface relief variation, we consider a grayscale relief map. This conversion is done by two parameters: intensity_per_cm and $\min R V$. The parameter $\min R V$ corresponds to the intensity 0 of the relief map for the smallest relief to represent. It is recommended to use a negative value for $\min R V$, this allows to represent the whole surface of the trunk. The parameter intensity_per_cm allows to define the number of gray levels to represent $1 \mathrm{~cm}$ of relief. The choice of the value of intensity_per_cm should not be too high because it determines the maximum representable relief. If intensity_per_cm $=50$, the relief map will be able to represent at most a relief about $5 \mathrm{~cm}$. The value must not be chosen too low either because some singularities have a very low relief, for example the singularity named "picots" are not more than $1 \mathrm{~cm}$ high. By default, intensity_per_cm $=10$ and $\min R V=-5$ for all the relief maps generated for the training or for the tests of the segmentation method.

\subsection{Angular Division Parameter: Pad}

The width of the relief map corresponds to the maximum arc length of the mesh. To estimate it, we use an array of size Pad (see Section 3.1.2). The smaller the Pad, the more error will be made on the circumference of the trunk. For our data, we set $P a d=200$ by default.

\subsection{Scale Influence}

It should be mentioned that the trunks in our database present comparable scale size, with handannotated singularities (see Section 4.1). In order to evaluate the robustness of our method with respect to the mesh scale, we have changed the scale of the WildCherry1 mesh using the following values: $0.8,1.2,1.5,3,5$. This experiment is done for the purpose of verifying whether our method is able to perform the singularity segmentation on larger trunks. Figure 18 shows the relief maps generated from the enlarged mesh and the associated segmentation. The relief map seems to be impacted by the scale factor. The perturbation of the relief map by the scale change impacts the quality of the segmentation. We think that this is due to the fixed size of the rectangular patch used to calculate the delta distance (see Section 3.1.1). Therefore, it would be necessary to automatically adapt the rectangular patch size with respect to the studied trunk. Moreover, the relief maps used to train our models are generated from trunk meshes of similar size. It would be interesting to study and apply a scale variation on the training data to allow the network to generalize the segmentation on larger trunks. However, scale changes by a factor of 5 can be considered as very special cases which are infrequent for usual application cases.

\section{Conclusion}

In this article, we proposed a method allowing to automatically segment the singularities on the tree bark surface. It is adapted from [1]. More precisely, the method is based on the construction of a relief map able to locally adapt itself to the global shape of the trunk, and combines with a convolutional neural network to improve the resulting segmentation quality. Furthermore, we bring an improvement in generating relief maps compared to [1]. This improvement allows to build relief maps from partial meshes of trunks where a cylindrical sector may be missing. Moreover, we detail the algorithms and the code used to generate the relief map. The cross validation shows that the training is robust to our database (the standard deviation is low). However, we believe that our segmentation method would benefit from using more 3D information. 

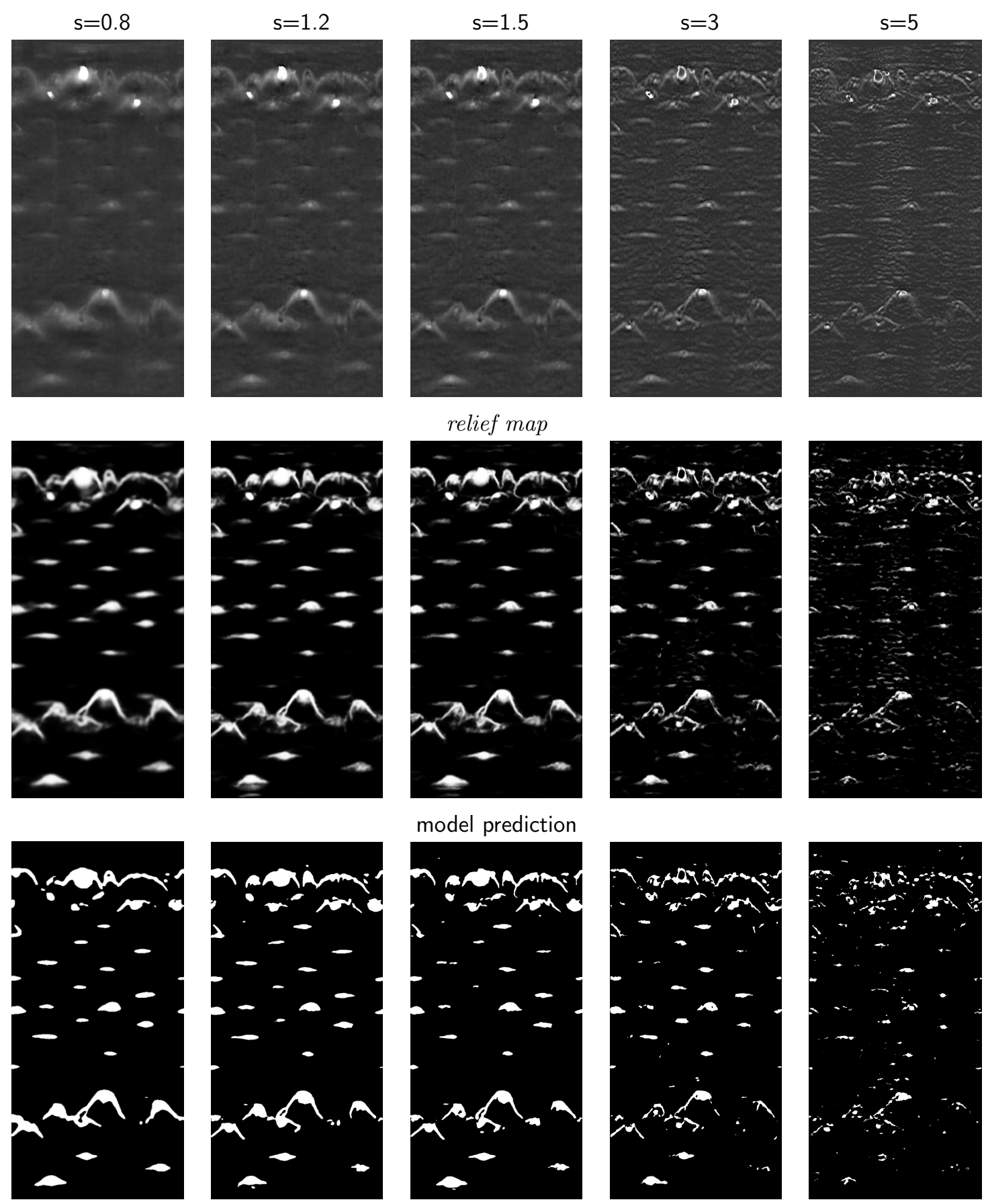

model prediction
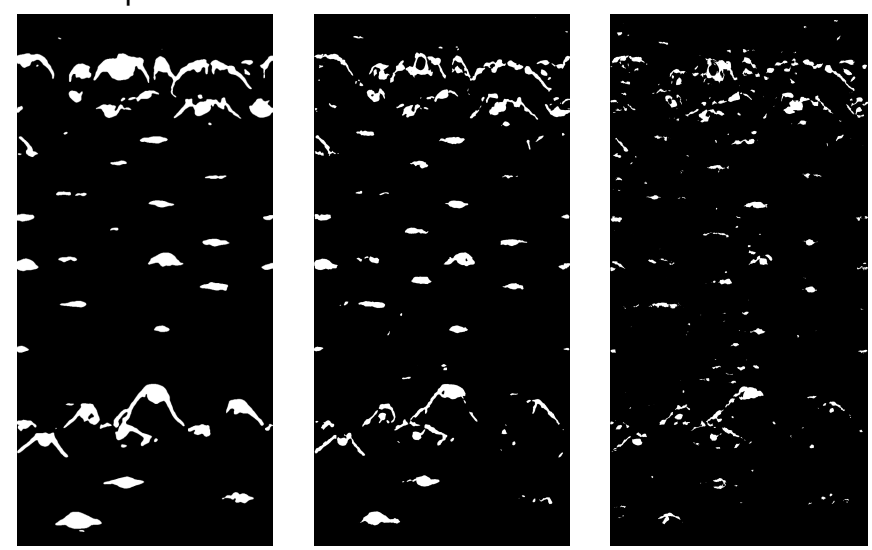

model prediction $(\mathrm{t}=0.5)$

Figure 18: Our method segmentation on the example WildCherry1 at different scales.

In the future, we would like to work on the generation of a new type of map from the trunk meshes, in particular using the normals from face meshes. It will allow to represent the orientation of the face meshes in the image. We then will train a CNN to accumulate orientation and relief information to produce a segmentation. In addition, we plan to address the related objective of classifying the singularities according to their different types. Such features are interesting to get a finer estimation of the wood quality. 


\section{Acknowledgment}

This research was made possible with the support from the French National Research Agency, in the framework of the project WoodSeer, ANR-19-CE10-011.

\section{Image Credits}

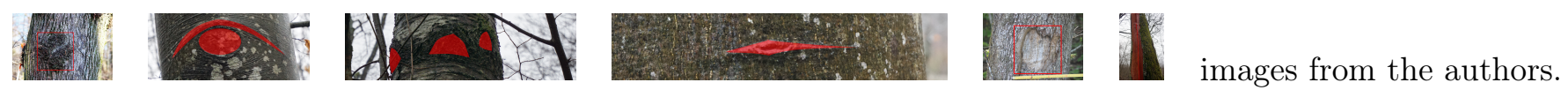

\section{References}

[1] F. Delconte, P. Ngo, I. Debled-Rennesson, B. Kerautret, V-T. Nguyen, And T. Constant, Tree Defect Segmentation using Geometric Features and CNN, in Reproducible Research on Pattern Recognition (RRPR), 2021. https://doi.org/10.1007/ 978-3-030-76423-4_6.

[2] T. Devries And G.W. TAYLOR, Improved regularization of convolutional neural networks with cutout, ArXiv preprint, abs/1708.04552 (2017). https://arxiv.org/abs/1708.04552.

[3] N. IbtehaZ And M.S. Rahman, MultiResUNet: Rethinking the U-Net architecture for multimodal biomedical image segmentation, Neural Networks, 121 (2020), pp. 74-87. https: //doi.org/10.1016/j.neunet.2019.08.025.

[4] V. Iglovikov And A. Shvets, Ternausnet: U-net with VGG11 encoder pre-trained on imagenet for image segmentation, arXiv preprint, (2018). https://arxiv.org/abs/1801.05746.

[5] B. Kerautret, A. Krähenbühl, I. Debled-Rennesson, and J. O. Lachaud, Centerline detection on partial mesh scans by confidence vote in accumulation map, in International Conference on Pattern Recognition (ICPR), 2016, pp. 1376-1381. http://dx.doi.org/10.1109/ ICPR. 2016.7899829.

[6] - On the Implementation of Centerline Extraction based on Confidence Vote in Accumulation Map, in International Workshop on Reproducible Research in Pattern Recognition, B. Kerautret, M. Colom, and P. Monasse, eds., vol. 10214, Springer, 2016, pp. 109-123. http://dx.doi.org/ 10.1007/978-3-319-56414-2_9.

[7] D.P. Kingma And J. BA, Adam: A Method for Stochastic Optimization, in International Conference on Learning Representations, 2014.

[8] U. Kretschmer, N. Kirchner, C. Morhart, and H. Spiecker, A new approach to assessing tree stem quality characteristics using terrestrial laser scans, Silva Fennica, 47 (2013), p. 1071. http://dx.doi.org/10.14214/sf.1071.

[9] L. Lu, Y. Shin, Y. Su, And G. Em Karniadakis, Dying ReLU and Initialization: Theory and Numerical Examples, Communications in Computational Physics, 28 (2020), pp. 1671-1706. https://doi .org/10.4208/cicp.0A-2020-0165.

[10] V-T. Nguyen, T. Constant, B. Kerautret, I. Debled-Rennesson, and F. Colin, A machine-learning approach for classifying defects on tree trunks using terrestrial LiDAR, 
Computers and Electronics in Agriculture, 171 (2020), p. 105332. https://doi.org/10.1016/ j.compag.2020.105332.

[11] V-T. Nguyen, B. Kerautret, I. Debled-Rennesson, F. Colin, A. Piboule, And T. Constant, Segmentation of defects on log surface from terrestrial lidar data, in International Conference on Pattern Recognition (ICPR), IEEE, 2016, pp. 3168-3173. https: //doi.org/10.1109/ICPR.2016.7900122.

[12] — Algorithms and implementation for segmenting tree log surface defects, in International Workshop on Reproducible Research in Pattern Recognition, B. Kerautret, M. Colom, and P. Monasse, eds., Cham, 2017, Springer International Publishing, pp. 150-166. http://dx. doi .org/10.1007/978-3-319-56414-2_11.

[13] G. Pan, Y. Zheng, S. Guo, And Y. Lv, Automatic sewer pipe defect semantic segmentation based on improved U-Net, Automation in Construction, 119 (2020), p. 103383. https://doi. $\mathrm{org} / 10.1016 / \mathrm{j}$. autcon.2020.103383.

[14] O. Ronneberger, P. Fischer, And T. Brox, U-net: Convolutional networks for biomedical image segmentation, in International Conference on Medical image computing and computer-assisted intervention, Springer, 2015, pp. 234-241. https://doi.org/10.1007/ 978-3-319-24574-4_28.

[15] — U-Net: Convolutional Networks for Biomedical Image Segmentation, in Medical Image Computing and Computer-Assisted Intervention (MICCAI), 2015. https://doi .org/10.1007/ 978-3-319-24574-4_28.

[16] P. Rosin, Unimodal thresholding, Pattern Recognition, 34 (2001), pp. 2083-2096. http://dx. doi.org/10.1016/S0031-3203(00)00136-9.

[17] D. Tabernik, S. Šela, J. SkvarČ, And D. SkoČAJ, Segmentation-based deep-learning approach for surface-defect detection, Journal of Intelligent Manufacturing, 31 (2020), pp. 759-776. https://doi.org/10.1007/s10845-019-01476-x.

[18] F.H. Wagner, A. Sanchez, Y. Tarabalka, R.G. Lotte, M.P. Ferreira, M.P.M. Aidar, E. Gloor, O.L. Phillips, And L.E.O.C. AragaO, Using the U-net convolutional network to map forest types and disturbance in the Atlantic rainforest with very high resolution images, Remote Sensing in Ecology and Conservation, 5 (2019), pp. 360-375. https: //doi.org/10.1002/rse2.111.

[19] X-Y. Zhou And G-Z. YAng, Normalization in training U-Net for 2-D biomedical semantic segmentation, IEEE Robotics and Automation Letters, 4 (2019), pp. 1792-1799. https://doi. org/10.1109/LRA.2019.2896518.

[20] Z. Zhou, M.M.R. Siddiquee, N. Tajbakhsh, and J. Liang, Unet++: A nested U-Net architecture for medical image segmentation, in Deep Learning in Medical Image Analysis and Multimodal Learning for Clinical Decision Support, Springer, 2018, pp. 3-11. https://doi. org/10 .1007/978-3-030-00889-5_1. 